\title{
染料木素氨基甲酸酯类衍生物的合成及生物活性研究
}

\author{
强晓明 ${ }^{a}$ 袁 文 $^{a}$ 桑志培 ${ }^{a}$ 邓 勇*,a,b \\ $\left({ }^{a}\right.$ 四川大学华西药学院药物化学系 成都 610041) \\ $\left({ }^{b}\right.$ 四川大学华西药学院 靶向药物及释药系统教育部重点实验室 成都 610041)
}

\begin{abstract}
摘要 基于多靶点药物设计策略, 以染料木素为先导化合物, 对其 7-位和 4'-位差基进行修饰, 合成了 24 个染料木素氨 基甲酸酯类衍生物, 其化学结构经 ${ }^{1} \mathrm{H}$ NMR 和 HRMS 确证. 生物活性测试结果表明, 部分化合物对乙酰胆碱酷酶具有 较强抑制活性, 且对 $\mathrm{H}_{2} \mathrm{O}_{2}$ 诱导的 PC12 细胞氧化损伤具有显著保护作用.

关键词 阿尔茨海默症; 多靶点药物治疗; 染料木素氨基甲酸酯类衍生物; 合成; 乙酰胆碱酯酶抑制剂; 神经保护剂
\end{abstract}

\section{Synthesis and Biological Evaluation of Genistein Carbamate Derivatives}

\author{
Qiang, Xiaoming ${ }^{a} \quad{\text { Yuan, } \text { Wen }^{a} \quad \text { Sang, Zhipei }}^{a} \quad$ Deng, Yong ${ }^{*, a, b}$ \\ ( ${ }^{a}$ Department of Medicinal Chemistry, West China School of Pharmacy, Sichuan University, Chengdu 610041) \\ $\left({ }^{b}\right.$ Key Laboratory of Drug Targeting and Drug Delivery System, Ministry of Education, \\ West China School of Pharmacy, Sichuan University, Chengdu 610041)
}

\begin{abstract}
With genistein as lead compound, twenty-four genistein carbamate derivatives were synthesized from genistein with the structural modification of $7-\mathrm{OH}$ and $4^{\prime}-\mathrm{OH}$ based on the muti-target-directed drug design strategy. The chemical structures of target compounds were confirmed by ${ }^{1} \mathrm{H}$ NMR and HRMS techniques. Their acetylcholinesterase and butylcholinesterase inhibitory activity and neuroprotective effects against hydrogen peroxide induced PC12 cell injury were evaluated in vitro. The results indicated that some compounds exhibited the most potent acetylcholinesterase inhibitory activity and neuroprotective effects.
\end{abstract}

Keywords alzheimer's disease; muti-target therapeutics; genistein carbamate derivatives; synthesis; AChE inhibitors; neuroprotective agents

阿尔茨海默症(alzheimer's disease, AD)亦称老年痴 呆(senile dementia), 是一种以进行性认知功能障碍和记 忆力损害为主要特征的中枢神经系统退行性疾病, 其发 病率在 65 岁以上老年人中约为 $10 \%, 85$ 岁以上约为 $47 \%$, 在我国老年痴呆患者人数约 $600 \sim 700$ 万, 发病率 超过 $5 \%{ }^{[1]}$. 随着全球人口老龄化进程的加快, 其发病率 呈明显上升趋势, $\mathrm{AD}$ 已成为严重威胁人类生活和健康 的重大疾病之一. 目前, 已批准用于治疗 $\mathrm{AD}$ 的药物主 要为乙酰胆碱酯酶(AchE)抑制剂, 包括他克林(tacrine)、 多奈哌齐(donepezil)、卡巴拉汀(rivastigmine)、加兰他敏 (galantamine)和石杉碱甲(huperzine A) 等, 临床试验表 明, 该类药物可通过提高患者体内乙酰胆碱水平有效缓
解 $\mathrm{AD}$ 症状, 但不能有效阻止或逆转病程，而且还会引 起头晕、恶心、食欲不振以及大便频繁等副作用，因而 长期疗效不甚理想 ${ }^{[2]}$. 因此, 发现新型作用机制的 $\mathrm{AD}$ 治疗药物具有重要现实意义.

研究表明, $\mathrm{AD}$ 的发生和发展是多种分子机制、多种 因素相互作用的结果, 采用基于 “多靶点药物治疗” 策 略已成为抗 $\mathrm{AD}$ 药物研究的重要方向. 由于 $\mathrm{AD}$ 的发病 机制尚未完全阐明，但患者中枢胆碱水平下降、 $\beta$-淀粉 样蛋白 $(\beta$-Amyloid peptide, $\mathrm{A} \beta)$ 的过度生成与沉积以及 氧化应激损伤在 $\mathrm{AD}$ 发病过程中起着重要的作用 ${ }^{[3]}$, 因 此可以将胆碱酯酶抑制剂与抗氧化剂组合, 通过抑制乙 酰胆碱酯酶(acetylcho linesterose, AChE)的活性以及清

\footnotetext{
*E-mail: dengyongy@sohu.com

Received October 22, 2012; revised November 10, 2012; published online November 23, 2012.

Project supported by the National Natural Science Foundation of China (No. 20672077, 20872099), the Research Fund for the Doctoral Program of Higher Education (No. 20110181110079) and the National Science and Technology Major Project (No. 2013ZX09102-012).

国家自然科学基金(Nos. 20672077, 20872099)、教育部博士点基金(No. 20110181110079)和国家科技重大专项(No. 2013ZX09301304-002)资助项目.
} 
除自由基来达到协同治疗 $\mathrm{AD}$ 的目的 ${ }^{[4]}$.<smiles>Nc1c2c(nc3ccccc13)CCCC2</smiles>

Tacrine
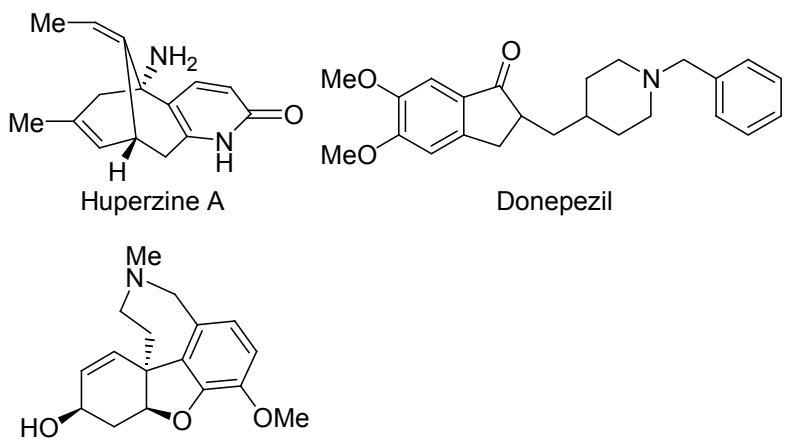

Galantamine

染料木素 $\left(5,7,4^{\prime}\right.$-三差基异黄酮, genistein, 1)又名金 雀异黄素, 是自然界广泛存在的植物雌激素类异黄酮, 药效学研究表明, 染料木素具有抗氧化、改善学习和记 忆的功能, 是治疗 $\mathrm{AD}$ 等神经退行性疾病的较理想䧳激 素替代药物 ${ }^{[5]}$. 但染料木素的亲脂性、亲水性均较弱, 且
存在显著首过效应，导致其生物利用度低、体内半衰期 短，另外，该化合物极性较大，难以透过血脑屏障进入 中枢系统 ${ }^{[6]}$. 为此, 我们设想将卡巴拉汀的抗胆碱酷酶 药效基团一一氨基甲酸酯片段与染料木素拼合，设计并 合成一类染料木素氨基甲酸酯类杂合物(图 1 中 6,7 和 8 所示), 希望能从增强中枢乙酰胆碱能神经功能、抗自由 基和神经保护等多靶点作用达到协同治疗 $\mathrm{AD}$ 等神经退 行性疾病的作用. 初步体外生物活性篎选结果表明, 所 合成的部分目标物具有较强的 AchE 抑制活性，同时对 $\mathrm{H}_{2} \mathrm{O}_{2}$ 诱导的 PC12 细胞氧化损伤具有显著保护作用.

\section{1 结果与讨论}

\section{1 化合物的合成}

以染料木素(1)为起始原料，先用甲氧基甲醚对 1 的 7-羊基进行保护，与相应的氨基甲酰氯(3)反应后，再进 一步脱除甲氧基甲醚保护基，得染料木素-4'-氨基甲酸 酯类化合物 $6 \mathrm{a} \sim 6 \mathrm{~h}$; 利用 1 直接与相应的 3 反应，通过 控制 3 的用量和反应条件, 即可制备得到染料木素-7-氨 基甲酸酯类化合物 $7 \mathbf{a} \sim 8 \mathrm{~h}$ 和染料木素-7,4'-二氨基甲酸 酯类化合物 $\mathbf{8 a} \sim 8 \mathrm{~h}$ ，其合成路线如图 1 所示.

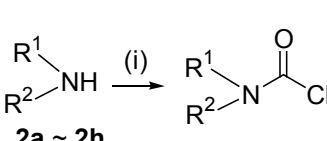

$$
\begin{aligned}
& 3 a \sim 3 h
\end{aligned}
$$<smiles>O=c1c(-c2ccc(O)cc2)coc2cc(O)cc(O)c12</smiles>

(v)

(ii)<smiles>COCOc1cc(O)c2c(=O)c(-c3ccc(O)cc3)coc2c1</smiles>

(iii)<smiles>[R]NC(=O)Oc1ccc(-c2coc3cc(OCOC)cc(O)c3c2=O)cc1</smiles>

(iv)<smiles>[R1]N([R])C(=O)Oc1ccc(-c2coc3cc(O)cc(O)c3c2=O)cc1</smiles>

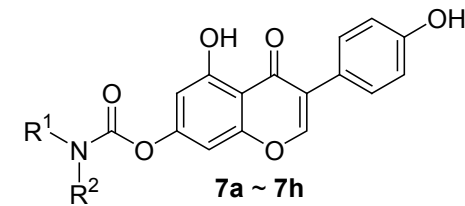<smiles>[R1]N([R])C(=O)Oc1ccc(-c2coc3cc(OC(=O)N([R])[R])cc(O)c3c2=O)cc1</smiles>

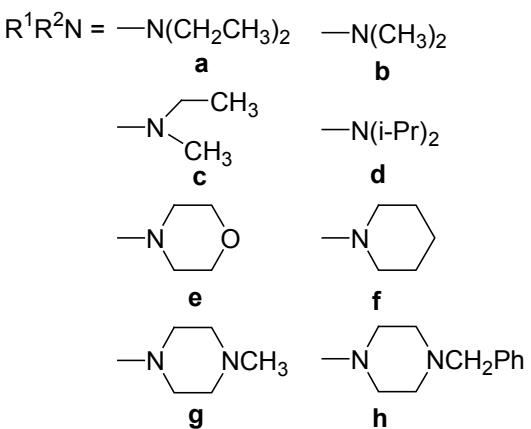

Reagents and conditions: (i) triphosgene, $\mathrm{NaHCO}_{3}, \mathrm{CH}_{2} \mathrm{Cl},-10 \sim 0{ }^{\circ} \mathrm{C}, 6 \sim 8 \mathrm{~h}$; (ii) $\mathrm{CH}_{3} \mathrm{OCH}_{2} \mathrm{Cl}, \mathrm{KOH}$, DMF, r.t. $12 \mathrm{~h}$; (iii) $\mathrm{R}{ }^{1} \mathrm{R}^{2} \mathrm{NCOCl}$ (3a 3h), $\mathrm{K}_{2} \mathrm{CO}_{3}, \mathrm{CH}_{3} \mathrm{CN}, 60 \sim 65{ }^{\circ} \mathrm{C}, 6 \sim 10 \mathrm{~h}$; (iv) $10 \% \mathrm{HCl}, \mathrm{C}_{2} \mathrm{H}_{5} \mathrm{OH}$, reflux, $3 \mathrm{~h}$; (v) $\mathrm{R}^{1} \mathrm{R}^{2} \mathrm{NCOCl}$ (3a $\sim 3 \mathrm{~h}$ ), $\mathrm{KOH}$, DMF, r.t., $12 \sim 15$ h; (vi) $\mathrm{R}^{1} \mathrm{R}^{2} \mathrm{NCOCl}(\mathbf{3 a} \sim 3 \mathrm{~h}), \mathrm{K}_{2} \mathrm{CO}_{3}$, acetone, reflux, 12 15 h.

图 1 化合物 6,7 和 8 的合成路线

Figure 1 The synthetic route of compounds 6, 7 and 8 
在染料木素分子中存在 3 个酚羟基, 其中 5-羟基因 与 4-羰基形成分子内氢键, 较难发生酰化反应, 7-羊基 的酸性较 4'-羟基强 ${ }^{[7]}$, 在碱性条件下, 染料木素 7-羟基 易形成酚氧负离子, 显著增强其亲核性, 从而可选择性 地被氨基甲酰氯(3)酰化, 得到染料木素-7-氨基甲酸酯 类化合物 $7 \mathbf{a} \sim 7 \mathbf{h}$, 试验过程中我们发现, 采用 $\mathrm{K}_{2} \mathrm{CO}_{3} /$ 丙酮回流反应条件时 ${ }^{[8]}$, 当化合物 $\mathbf{3}$ 与 $\mathbf{1}$ 的物质的量比 为 $1.2: 1$ 时, 虽生成主成分为所需的 7 外, 但还有相当 数量的 $\mathbf{6}$ 和 8 生成, 化合物 $\mathbf{6}$ 与 7 极性相似, 无法通过 柱层析分离纯化. 我们参考文献[7]以 $\mathrm{DMF}$ 为溶剂, 先 用等物质的量的 $\mathrm{KOH}$ 与染料木素反应形成 7 位酚钾盐, 然后在室温条件下与相应的 $\mathbf{3}$ 反应, 经硅胶柱层析纯化 即可制备得到相应的 7 , 反应过程中除生成少量的双酰 化产物 8 外, 未发现有化合物 $\mathbf{6}$ 生成.

对于目标物 6 的合成, 考虑到用 1 直接酰化存在选 择性低、分离纯化困难等问题, 我们选择先以甲氧基甲 醚对化合物 1 的 7-羟基进行保护, 再对其 4'位羟基进行 酰化的合成策略, 在合成 7-甲氧甲氧基-5,4'一二羟基异黄 酩(4)的过程中, 我们发现, 采用 $\mathrm{K}_{2} \mathrm{CO}_{3}$ /丙酩回流反应 条件下与氯甲基甲醚反应 ${ }^{[8]}$, 除主要生成所需的 $\mathbf{4}$ 外, 还有相当数量的 $4^{\prime}$ 烃化和 $7,4^{\prime}$ 双烃化产物生成, 为此, 我们采用上述制备 7 的反应条件, 成功获得 4 , 反应收 率由文献[8]报道的 $19.0 \%$ 提高到 $61.5 \%$, 且粗品只需经 简单的重结晶即可纯化, 避免使用文献报道的柱层析分 离. 对于目标物 8 的合成, 我们采用常规反应条件, 以 $\mathrm{K}_{2} \mathrm{CO}_{3} /$ 丙酮回流反应条件下与过量的 3 反应, 即可制得 相应目标物.

\section{2 化合物的生物活性筛选}

采用改进的 Ellman ${ }^{[9]}$ 法, 我们测定了所有目标物对 乙酰胆碱酯酶( $\mathrm{AChE}$ )和丁酰胆碱酯酶(BuChE)的抑制活 性, 以重酒石酸卡巴拉汀(自制, 纯度大于 $99.5 \%$ )为阳 性对照. 对乙酰胆碱酷酶具有较强活性的部分化合物, 我们还利用 $\mathrm{H}_{2} \mathrm{O}_{2}$ 诱导的 $\mathrm{PC} 12$ 细胞氧化损伤模型 ${ }^{[10]}$ 测 试了该类化合物对氧化应激所致神经细胞损伤的保护 作用, 该篎选模型以染料木素为阳性对照. 其生物活性 测试结果如表 1 所示.

对 $\mathrm{AChE}$ 抑制活性测试结果表明, 在 $5.0 \mu \mathrm{mol} / \mathrm{L}$ 浓 度下, 染料木素(1)对胆碱酯酶无抑制活性, 而本文所合 成的染料木素氨基甲酸酯类衍生物对 $\mathrm{AChE}$ 显示强度不 等的抑制活性, 表明氨基甲酸酯结构单元为此类目标物 具有 AChE 抑制活性的药效基团; 其中, 染料木素-4'-氨 基甲酸酯类化合物 $6 \mathbf{a} \sim 6 \mathrm{~h}$ 和染料木素-7-氨基甲酸酯类 化合物 7a $\sim 7 \mathbf{h}$ 对 $\mathrm{AChE}$ 的抑制活性均低于对照药重酒 石酸卡巴拉汀 (抑制率为 $47.5 \%, \mathrm{IC}_{50}$ 为 $6.3 \mu \mathrm{mol} / \mathrm{L}$ ); 部
分染料木素-7,4'-二氨基甲酸酯类化合物 $8 \mathbf{a} \sim \mathbf{8 h}$ 对 $\mathrm{AChE}$ 具有较好的抑制活性, 其中化合物 $\mathbf{8 g}$ (抑制率为 $64.9 \%, \mathrm{IC}_{50}$ 为 $1.2 \mu \mathrm{mol} / \mathrm{L}$ )的活性显著高于重酒石酸卡巴 拉汀, 化合物 $8 \mathrm{e}$ (抑制率为 $41.2 \%$ )的活性接近重酒石酸 卡巴拉汀. 上述结果还表明, 在氨基甲酸酯结构单元中, 含 4-甲基哌嗪的目标物 $6 \mathrm{~g}, 7 \mathrm{~g}, 8 \mathrm{~g}$ 对 $\mathrm{AChE}$ 的抑制活性 均高于含 4-茮基哌嗪目标物 $6 \mathbf{h}, 7 \mathbf{h}, 8 \mathrm{~h}$; 含 7,4'-双吗啉 基或 4-甲基哌嗪结构的目标物 $8 \mathrm{e}$ 或 $8 \mathrm{~g}$ 活性高于含单吗 啉基或 4-甲基哌嗪结构的目标物 $6 \mathrm{e}, 7 \mathrm{e}$ 或 $6 \mathrm{~g}, 7 \mathrm{~g}$. 对 $\mathrm{BuChE}$ 的抑制活性测试结果表明, 在 $5.0 \mu \mathrm{mol} / \mathrm{L}$ 浓度下, 所有目标物对 $\mathrm{BuChE}$ 均无抑制作用，而重酒石酸卡巴 拉汀对 $\mathrm{BuChE}$ 具有较强抑制活性(抑制率为 $68.2 \%, \mathrm{IC}_{50}$ 为 $1.37 \mu \mathrm{mol} / \mathrm{L}$ ), 说明该类目标物对 $\mathrm{AChE}$ 的抑制作用 具有一定选择性，可在一定程度上克服重酒石酸卡巴拉 汀对外周神经系统的 BuChE 抑制所导致的毒副作用.

神经细胞损伤理论认为, 氧化应激是神经细胞损害 的主要分子机制, 在氧化应激过程中会产生包括: 超氧 阴离子 $\left(\mathrm{O}^{2-}\right)$ 、过氧化氢 $\left(\mathrm{H}_{2} \mathrm{O}_{2}\right)$ 、羟基游离基 $(\cdot \mathrm{OH})$ 和单 线态氧 $\left({ }^{1} \mathrm{O}_{2}\right)$ 等活性氧自由基, 从而引起细胞脂质过氧 化、损伤 DNA 分子、调节细胞调亡相关基因等导致神 经细胞调亡 ${ }^{[3]}$. 其中, $\mathrm{H}_{2} \mathrm{O}_{2}$ 诱导的细胞调亡具有普遍性, 因此, 常利用 $\mathrm{H}_{2} \mathrm{O}_{2}$ 诱导的 PC12 细胞氧化损伤模型来测 试化合物对氧化应激所导致的神经细胞损伤的保护作 用. 本文利用上述氧化损伤模型测试了部分目标物的神 经保护活性, 测试结果表明, 在 $10.0 \mu \mathrm{mol} / \mathrm{L}$ 浓度下, 对 $\mathrm{AChE}$ 具有较强抑制作用的化合物对 $\mathrm{H}_{2} \mathrm{O}_{2}$ 诱导的 PC12 细胞氧化损伤均具有显著保护作用, 其抗氧化活性强于 先导化合物染料木素, 且 7 或 4'-单取代氨基甲酸酯类化 合物 $6 \mathrm{~g}$ 或 $7 \mathrm{~g}$ 的抗氧化活性强于 7,4'-双取代氨基甲酸酯 类化合物 8g.

\section{2 结论}

基于多靶点药物设计策略, 以染料木素为先导化合 物, 分别对其 7-位和 4'-位羟基进行修饰, 设计并合成了 24 个染料木素氨基甲酸酯类衍生物, 并对目标物的碱 酷酶抑制活性和对 $\mathrm{H}_{2} \mathrm{O}_{2}$ 诱导的 PC12 细胞氧化损伤保护 作用进行了测试, 初步篎选结果表明, 所有目标物均无 抗 $\mathrm{BuChE}$ 活性, 部分化合物对 $\mathrm{AChE}$ 具有较强抑制作 用，其中化合物 $8 \mathrm{~g}$ 的 $\mathrm{AChE}$ 抑制活性强于阳性对照药重 酒石酸卡巴拉汀; 对 $\mathrm{AChE}$ 具有较强抑制作用的目标物 还对 $\mathrm{H}_{2} \mathrm{O}_{2}$ 诱导的 $\mathrm{PC} 12$ 细胞氧化损伤具有显著保护作 用. 该类化合物的构效关系和进一步生物活性评价仍在 进行中. 
表 1 染料木素氨基甲酸酯类化合物对胆碱酯酶的抑制活性和神经保护作用 ${ }^{a}$

Table 1 Inhibition of $\mathrm{AChE}$ and BuChE activities and neuroprotective effects of genistein carbamates

\begin{tabular}{|c|c|c|c|c|c|}
\hline \multirow{2}{*}{ Compound } & \multicolumn{2}{|c|}{$\mathrm{AChE}$} & \multicolumn{2}{|c|}{$\mathrm{BuChE}$} & \multirow{2}{*}{$\frac{\text { Neuroprotective effect }^{c}}{\text { Cell viability } / \%}$} \\
\hline & Inhibition $^{b} / \%$ & $\mathrm{IC}_{50} /\left(\mu \mathrm{mol} \cdot \mathrm{L}^{-1}\right)$ & Inhibition $^{b} / \%$ & $\mathrm{IC}_{50} /\left(\mu \mathrm{mol} \cdot \mathrm{L}^{-1}\right)$ & \\
\hline 1 & $<5.0$ & $>50$ & $<5.0$ & N.T. ${ }^{d}$ & $69.48 \pm 0.30$ \\
\hline $6 a$ & 20.4 & $>50$ & $<5.0$ & N.T. & $78.68 \pm 0.71$ \\
\hline $6 \mathbf{b}$ & 13.8 & $>50$ & $<5.0$ & N.T. & $77.45 \pm 0.49$ \\
\hline $6 c$ & 9.2 & $>50$ & $<5.0$ & N.T. & N.T. \\
\hline 6d & 8.6 & $>50$ & $<5.0$ & N.T. & N.T. \\
\hline $6 e$ & 10.4 & $>50$ & $<5.0$ & N.T. & N.T. \\
\hline $6 f$ & 14.7 & $>50$ & $<5.0$ & N.T. & $75.06 \pm 0.42$ \\
\hline $6 \mathrm{~g}$ & 18.1 & $>50$ & $<5.0$ & N.T. & $84.78 \pm 1.06$ \\
\hline $6 \mathrm{~h}$ & 9.9 & $>50$ & $<5.0$ & N.T. & N.T. \\
\hline $7 a$ & 7.9 & $>50$ & $<5.0$ & N.T. & N.T. \\
\hline $7 b$ & 8.8 & $>50$ & $<5.0$ & N.T. & N.T. \\
\hline $7 c$ & 17.7 & $>50$ & $<5.0$ & N.T. & $77.18 \pm 1.31$ \\
\hline $7 d$ & 8.5 & $>50$ & $<5.0$ & N.T. & N.T. \\
\hline $7 e$ & 8.2 & $>50$ & $<5.0$ & N.T. & N.T. \\
\hline $7 f$ & 5.5 & $>50$ & $<5.0$ & N.T. & N.T. \\
\hline $7 \mathrm{~g}$ & 36.1 & 22.0 & $<5.0$ & N.T. & $85.10 \pm 1.72$ \\
\hline $7 \mathrm{~h}$ & 23.9 & $>50$ & $<5.0$ & N.T. & $81.89 \pm 0.72$ \\
\hline $8 \mathbf{a}$ & 13.6 & $>50$ & $<5.0$ & N.T. & N.T. \\
\hline $8 \mathbf{b}$ & 10.6 & $>50$ & $<5.0$ & N.T. & N.T. \\
\hline $8 c$ & 9.3 & $>50$ & $<5.0$ & N.T. & N.T. \\
\hline $8 d$ & $<5.0$ & $>50$ & $<5.0$ & N.T. & N.T. \\
\hline $8 e$ & 41.2 & 11.0 & $<5.0$ & N.T. & $72.54 \pm 0.28$ \\
\hline $8 f$ & 8.9 & $>50$ & $<5.0$ & N.T. & N.T. \\
\hline $8 g$ & 64.9 & 1.2 & $<5.0$ & N.T. & $78.27 \pm 0.83$ \\
\hline $8 \mathbf{h}$ & 17.8 & $>50$ & $<5.0$ & N.T. & $78.42 \pm 0.80$ \\
\hline 重酒石酸卡巴拉汀 & 47.5 & 6.3 & 68.2 & 1.37 & N.T. \\
\hline
\end{tabular}

${ }^{a}$ All experiments performed in triplicate; ${ }^{b}$ Inhibition was determined at $5.0 \mu \mathrm{mol} / \mathrm{L}$ inhibitor concentration; ${ }^{c}$ The neuroprotective effects of compounds at $10 \mu \mathrm{mol} / \mathrm{L}$ on cell injury induced by hydrogen peroxide $(100 \mu \mathrm{mol} / \mathrm{L})$ in $\mathrm{PC} 12$ cells. The cell viability in control was taken as $100 \%$, and cell viability in hydrogen peroxide $(100$ $\mu \mathrm{mol} / \mathrm{L}$ ) condition was $57.58 \% ;{ }^{d}$ N.T. means no tested.

\section{3 实验部分}

\section{1 仪器与试剂}

TYR-3 熔点仪, 温度计未经校正; Varian INOVA400 核磁共振仪(溶剂为 $\mathrm{CDCl}_{3}$ 或 DMSO- $d_{6}$, 内标 TMS); Agilent-6210 TOF LC/MS 高分辨质谱仪. 薄层层析用硅 胶板为山东烟台化工研究所生产, 用紫外灯或碘显色. 染料木素 $(>98 \%$, 南京泽朗医药科技有限公司), 其余 试剂均为市售分析纯或化学纯, 并根据需要进行常规处 理.

\section{2 氨基甲酰氯( $3 a \sim 3 h)$ 的制备}

将三光气 $(6.0 \mathrm{~g}, 20.0 \mathrm{mmol})$ 、二氯甲烷 $(50 \mathrm{~mL})$ 和 $\mathrm{NaHCO}_{3}(5.24 \mathrm{~g}, 62.3 \mathrm{mmol})$ 加入反应瓶中, 置冰盐浴中 冷却至 $0{ }^{\circ} \mathrm{C}$ 以下, 滴加入有机胺 $\mathbf{2 a} \sim \mathbf{2 h}(30.0 \mathrm{mmol})$ 溶 于二氯甲烷 $(10 \mathrm{~mL})$ 的溶液, 控制反应液温度不超过 0 ${ }^{\circ} \mathrm{C}$, 滴毕, 自然升温至室温摚拌反应 $6 \sim 8 \mathrm{~h}$, 反应结束 后，抽滤，少量二氯甲烷洗涤滤饼，滤液及洗液合并后 减压蒸除溶剂, 残余物减压蒸馏或硅胶柱层析纯化 [流
动相: $V$ (二氯甲烷 $): V$ (石油醚) $=3: 1]$, 收集所需组分, 即可得到相应的氨基甲酰氯 $3 \mathbf{a} \sim 3 \mathbf{h}$, 其性状、产率及沸 点(熔点)数据如下:

$\mathrm{N}, \mathrm{N}$-二乙氨基甲酰氯(3a): 无色油状液体, 产率 $73.2 \%$, b.p. $70.0 \sim 72.5{ }^{\circ} \mathrm{C} / 3.4 \mathrm{kPa}\left[\right.$ 文献值 $^{[11]}$ : b.p. $60.0 \sim$ $\left.62.0{ }^{\circ} \mathrm{C} / 2.0 \mathrm{kPa}\right]$.

$\mathrm{N}, \mathrm{N}$-二甲氨基甲酰氯(3b): 无色油状液体, 产率 $50.4 \%$, b.p. $69.0 \sim 72.0^{\circ} \mathrm{C} / 3.4 \mathrm{kPa}\left[\right.$ 文献值 $^{[12]}$ : b.p. 64.0 $\left.{ }^{\circ} \mathrm{C} / 2.67 \mathrm{kPa}\right]$.

$\mathrm{N}$-甲基- $\mathrm{N}$-乙基氨基甲酰氯(3c): 无色油状液体, 产 率 76.0\%, b.p. $94.0 \sim 96.0{ }^{\circ} \mathrm{C} / 6.0 \mathrm{kPa}\left[\right.$ 文献值 $^{[11]}$ : b.p. 88 $\left.89{ }^{\circ} \mathrm{C} / 5.33 \mathrm{kPa}\right]$.

$N, N$-二异丙氨基甲酰氯 (3d): 白色固体, 产率 $45.6 \%$, b.p. $120.0 \sim 122.5{ }^{\circ} \mathrm{C} / 2.0 \mathrm{kPa}$, m.p. $52.0 \sim 54.0{ }^{\circ} \mathrm{C}$ [文献值 ${ }^{[13]}$ : m.p. $55.0{ }^{\circ} \mathrm{C}$ ].

吗啡啉- $N$-甲酰氯(3e): 无色油状液体, 产率 $75.3 \%$, b.p. $114 \sim 116{ }^{\circ} \mathrm{C} / 2.0 \mathrm{kPa}$ [文献值 ${ }^{[14]}$ : b.p. $113{ }^{\circ} \mathrm{C} / 2.0 \mathrm{kPa}$ ].

哌啶- $N$-甲酰氯(3f): 无色油状液体，产率 $50.5 \%$, b.p. $118 \sim 120^{\circ} \mathrm{C} / 2.0 \mathrm{kPa}\left[\right.$ 文献值 ${ }^{[1]]}$ : b.p. $108 \sim 109$ 
$\left.{ }^{\circ} \mathrm{C} / 1.34 \mathrm{kPa}\right]$.

4-甲基哌嗪- $N$-甲酰氯(3g): 无色油状液体，产率 $62.0^{\circ}$, b.p. $122 \sim 124^{\circ} \mathrm{C} / 2.0 \mathrm{kPa}$ [文献值 ${ }^{[15]}$ : b.p. $84 \sim$ $\left.85^{\circ} \mathrm{C} / 0.4 \mathrm{kPa}\right]$.

4- 苄基哌嗪- $N$-甲酰氯(3h): 类白色固体，产率 $45.6 \%$, m.p. $90 \sim 92{ }^{\circ} \mathrm{C}$ [文献值 ${ }^{[16]}$ : m.p. $92{ }^{\circ} \mathrm{C}$ ].

\subsection{7-甲氧甲氧基-5,4'-二羟基异黄酮(4)的制备}

在反应瓶中加入染料木素 $\mathbf{1}(5.0 \mathrm{~g}, 18.5 \mathrm{mmol})$, $\mathrm{KOH}(1.1 \mathrm{~g}, 18.5 \mathrm{mmol})$, 碘化钾 $(0.3 \mathrm{~g}, 1.85 \mathrm{mmol})$ 和 $N, N$-二甲基甲酰胺 $(40 \mathrm{~mL})$, 室温摚拌反应 $1 \mathrm{~h}$ 后滴加入 氯甲基甲醚 $(1.68 \mathrm{~mL}, 22.2 \mathrm{mmol})$, 继续室温摚拌反应 12 $\mathrm{h}$. 反应结束后, 将反应液倒入去离子水 $(120 \mathrm{~mL})$ 中, 用 质量分数为 $10 \% \mathrm{HCl}$ 水溶液调节溶液 $\mathrm{pH}$ 至酸性, 过滤 析出的类白色固体, 所得粗品经乙醇重结晶, 得 $3.6 \mathrm{~g}$ 白 色固体, 收率 $61.5 \%$. m.p. $153 \sim 154{ }^{\circ} \mathrm{C}$ [文献值 ${ }^{[8]}$ : 收率 19.0\%, m.p. $151 \sim 154{ }^{\circ} \mathrm{C}$ ]. ${ }^{1} \mathrm{H}$ NMR (DMSO- $d_{6}, 400$ MHz) $\delta: 12.93$ (s, 1H, 5-OH), 8.32 (s, 1H, 2-H), 7.47 (d, $J$ $\left.=8.0 \mathrm{~Hz}, 2 \mathrm{H}, 2^{\prime}, 6^{\prime}-\mathrm{H}\right), 6.57(\mathrm{~d}, J=2.0 \mathrm{~Hz}, 1 \mathrm{H}, 8-\mathrm{H}), 6.38$ (d, $J=2.0 \mathrm{~Hz}, 1 \mathrm{H}, 6-\mathrm{H}), 6.87$ (d, $\left.J=8.0 \mathrm{~Hz}, 2 \mathrm{H}, 3^{\prime}, 5^{\prime}-\mathrm{H}\right)$, $5.21\left(\mathrm{~s}, 2 \mathrm{H}, \mathrm{OCH}_{2} \mathrm{O}\right), 3.42\left(\mathrm{~s}, 3 \mathrm{H}, \mathrm{OCH}_{3}\right)$.

\subsection{7-甲氧甲氧基-4'-氨基甲酰氧基-5-羟基异黄酮 (5a $\sim 5 h)$ 的制备}

将中间体 4 ( $0.20 \mathrm{~g}, 0.64 \mathrm{mmol})$ 、无水碳酸钾 $(0.14 \mathrm{~g}$, $0.96 \mathrm{mmol})$ 和乙腈 $(10 \mathrm{~mL})$ 加入反应瓶中, 搅拌均匀后加 入相应的氨基甲酰氯 $(3 \mathbf{a} \sim 3 \mathbf{h}) \quad(0.71 \mathrm{mmol})$, 升温至 $60 \sim 65{ }^{\circ} \mathrm{C}$ 搅拌反应 $6 \sim 10 \mathrm{~h}$, 反应结束后, 减压蒸除溶 剂, 残余物中加入去离子水 $(20 \mathrm{~mL})$, 用乙醚 $(25 \mathrm{~mL} \times 3)$ 萃取, 有机层合并后依次用质量分数为 $10 \% \mathrm{NaOH}$ 水溶 液 $(10 \mathrm{~mL} \times 2)$ 、去离子水 $(20 \mathrm{~mL})$ 和饱和氯化钠水溶液 $(20 \mathrm{~mL})$ 洗涤, 经无水硫酸钠干燥后过滤, 滤液减压蒸除 溶剂, 粗品经硅胶柱层析纯化 [流动相: $V$ (二氯甲烷) : $V$ (丙酮 $)=80: 1]$, 得 7-甲氧甲氧基-4'-氨基甲酰氧基-5羟基异黄酮 $(\mathbf{5} \mathbf{a} \sim \mathbf{5 h})$, 其性状、产率、熔点及波谱数据 如下.

7-甲氧甲氧基-4'-( $N, N$-二乙氨甲酰氧基)- 5 -羟基异 黄酮(5a): 浅黄色固体, 产率 78.9\%. m.p. $108 \sim 110{ }^{\circ} \mathrm{C}$; ${ }^{1} \mathrm{H}$ NMR $\left(\mathrm{CDCl}_{3}, 400 \mathrm{MHz}\right) \delta: 12.77$ (s, 1H, 5-OH), 7.90 (s, 1H, 2-H), 7.52 (d, $\left.J=8.4 \mathrm{~Hz}, 2 \mathrm{H}, 2^{\prime}, 66^{\prime}-\mathrm{H}\right), 7.20$ (d, $J=$ $\left.8.4 \mathrm{~Hz}, 2 \mathrm{H}, 3^{\prime}, 5^{\prime}-\mathrm{H}\right), 6.59$ (d, $\left.J=2.4 \mathrm{~Hz}, 1 \mathrm{H}, 6-\mathrm{H}\right), 6.51$ (d, $J=2.4 \mathrm{~Hz}, 1 \mathrm{H}, 8-\mathrm{H}), 5.24$ (s, $\left.2 \mathrm{H}, \mathrm{OCH}_{2} \mathrm{O}\right), 3.50$ (s, $3 \mathrm{H}$, $\mathrm{OCH}_{3}$ ), 3.40 (q, $J=6.8 \mathrm{~Hz}, 2 \mathrm{H}, \mathrm{NCH}_{2}$ ), 3.33 (q, $J=6.8$ $\mathrm{Hz}, 2 \mathrm{H}, \mathrm{NCH}_{2}$ ), 1.21 (t, $\left.J=6.8 \mathrm{~Hz}, 3 \mathrm{H}, \mathrm{CH}_{3}\right), 1.12$ (t, $J=$ $6.8 \mathrm{~Hz}, 3 \mathrm{H}, \mathrm{CH}_{3}$ ); MS (ESI) calcd for $\mathrm{C}_{22} \mathrm{H}_{24} \mathrm{NO}_{7}[\mathrm{M}+$ $\mathrm{H}]^{+}$414.1553, found 414.1558 .

7- 甲氧甲氧基-4'-( $N, N$-二甲氨甲酰氧基)-5-羊基异 黄酮(5b)：浅黄色固体，产率 $82.7 \%$. m.p. $166 \sim 168{ }^{\circ} \mathrm{C}$; ${ }^{1} \mathrm{H}$ NMR $\left(\mathrm{CDCl}_{3}, 400 \mathrm{MHz}\right) \delta: 12.77$ (s, 1H, 5-OH), 7.90 (s, 1H, 2-H), 7.52 (d, $\left.J=8.4 \mathrm{~Hz}, 2 \mathrm{H}, 2^{\prime}, 6^{\prime}-\mathrm{H}\right), 7.20$ (d, $J=$ $\left.8.4 \mathrm{~Hz}, 2 \mathrm{H}, 3^{\prime}, 5^{\prime}-\mathrm{H}\right), 6.59$ (d, $\left.J=2.4 \mathrm{~Hz}, 1 \mathrm{H}, 6-\mathrm{H}\right), 6.51$ (d, $J=2.4 \mathrm{~Hz}, 1 \mathrm{H}, 8-\mathrm{H}), 5.24\left(\mathrm{~s}, 2 \mathrm{H}, \mathrm{OCH}_{2} \mathrm{O}\right), 3.50(\mathrm{~s}, 3 \mathrm{H}$, $\mathrm{OCH}_{3}$ ), 3.12 (s, $3 \mathrm{H}, \mathrm{NCH}_{3}$ ), 3.03 (s, $3 \mathrm{H}, \mathrm{NCH}_{3}$ ); $\mathrm{MS}$ (ESI) calcd for $\mathrm{C}_{20} \mathrm{H}_{20} \mathrm{NO}_{7}[\mathrm{M}+\mathrm{H}]^{+}$386.1240, found 386.1232.

7-甲氧甲氧基-4'-( $N$-甲基- $N$-乙基氨甲酰氧基)-5-羊圣 基异黄酮(5c)：类白色固体，产率 55.0\%. m.p. 129 130 ${ }^{\circ} \mathrm{C} ;{ }^{1} \mathrm{H}$ NMR $\left(\mathrm{CDCl}_{3}, 400 \mathrm{MHz}\right) \delta$ : 12.77 (s, $\left.1 \mathrm{H}, 5-\mathrm{OH}\right)$, 7.90 (s, 1H, 2-H), 7.52 (d, $\left.J=8.4 \mathrm{~Hz}, 2 \mathrm{H}, 2^{\prime}, 6^{\prime}-\mathrm{H}\right), 7.19$ (d, $\left.J=8.4 \mathrm{~Hz}, 2 \mathrm{H}, 3^{\prime}, 5^{\prime}-\mathrm{H}\right), 6.58(\mathrm{~d}, J=2.0 \mathrm{~Hz}, 1 \mathrm{H}, 6-\mathrm{H}), 6.50$ (d, $J=2.0 \mathrm{~Hz}, 1 \mathrm{H}, 8-\mathrm{H}), 5.23\left(\mathrm{~s}, 2 \mathrm{H}, \mathrm{OCH}_{2} \mathrm{O}\right), 3.49$ (s, $3 \mathrm{H}$, $\left.\mathrm{OCH}_{3}\right), 3.41 \sim 3.48\left(\mathrm{~m}, 2 \mathrm{H}, \mathrm{NCH}_{2}\right), 3.08\left(\mathrm{~s}, 1.5 \mathrm{H}, \mathrm{NCH}_{3}\right)$, $3.00\left(\mathrm{~s}, 1.5 \mathrm{H}, \mathrm{NCH}_{3}\right), 1.18 \sim 1.27\left(\mathrm{~m}, 3 \mathrm{H}, \mathrm{CH}_{3}\right) ; \mathrm{MS}(\mathrm{ESI})$ calcd for $\mathrm{C}_{21} \mathrm{H}_{22} \mathrm{NO}_{7}[\mathrm{M}+\mathrm{H}]^{+}$400.14, found 400.1.

7-甲氧甲氧基-4'-( $N, N$-二异丙胺甲酰氧基)-5-羟基 异黄酮(5d): 浅黄色固体, 产率 53.6\%. m.p. 129 130 ${ }^{\circ} \mathrm{C} ;{ }^{1} \mathrm{H}$ NMR $\left(\mathrm{CDCl}_{3}, 400 \mathrm{MHz}\right) \delta$ : 12.78 (s, $\left.1 \mathrm{H}, 5-\mathrm{OH}\right)$, 7.89 (s, 1H, 2-H), 7.52 (d, $\left.J=8.4 \mathrm{~Hz}, 2 \mathrm{H}, 2^{\prime}, 6^{\prime}-\mathrm{H}\right), 7.20$ (d, $\left.J=8.4 \mathrm{~Hz}, 2 \mathrm{H}, 3^{\prime}, 5^{\prime}-\mathrm{H}\right), 6.58(\mathrm{~d}, J=2.0 \mathrm{~Hz}, 1 \mathrm{H}, 6-\mathrm{H}), 6.50$ (d, $J=2.0 \mathrm{~Hz}, 1 \mathrm{H}, 8-\mathrm{H}), 5.23$ (s, $2 \mathrm{H}, \mathrm{OCH}_{2} \mathrm{O}$ ), 4.12 (brs, $1 \mathrm{H}, \mathrm{NCH}$ ), 3.96 (brs, $1 \mathrm{H}, \mathrm{NCH}), 3.50$ (s, $\left.3 \mathrm{H}, \mathrm{OCH}_{3}\right), 1.30$ (brs, $12 \mathrm{H}, 4 \times \mathrm{CH}_{3}$ ); MS (ESI) calcd for $\mathrm{C}_{24} \mathrm{H}_{28} \mathrm{NO}_{7}[\mathrm{M}+$ $\mathrm{H}]^{+}$442.1866, found 442.1864 .

7-甲氧甲氧基-4'-(吗啡啉- $N$-甲酰氧基)-5-差基异黄 酮(5e): 类白色固体, 产率 $70.0 \%$. m.p. $176 \sim 177{ }^{\circ} \mathrm{C} ;{ }^{1} \mathrm{H}$ NMR (CDCl $3,400 \mathrm{MHz}) \delta: 12.74$ (s, 1H, 5-OH), 7.90 (s, $1 \mathrm{H}, 2-\mathrm{H}), 7.53$ (d, $\left.J=8.4 \mathrm{~Hz}, 2 \mathrm{H}, 2^{\prime}, 6^{\prime}-\mathrm{H}\right), 7.19$ (d, $J=8.4$ $\left.\mathrm{Hz}, 2 \mathrm{H}, 3^{\prime}, 5^{\prime}-\mathrm{H}\right), 6.58$ (d, $\left.J=1.6 \mathrm{~Hz}, 1 \mathrm{H}, 6-\mathrm{H}\right), 6.50$ (d, $J=$ $1.6 \mathrm{~Hz}, 1 \mathrm{H}, 8-\mathrm{H}), 5.23\left(\mathrm{~s}, 2 \mathrm{H}, \mathrm{OCH}_{2} \mathrm{O}\right), 3.75 \sim 3.77(\mathrm{~m}$, $4 \mathrm{H}, 2 \times \mathrm{OCH}_{2}$ ), 3.69 (brs, $2 \mathrm{H}, \mathrm{NCH}_{2}$ ) 3.58 (brs, $2 \mathrm{H}$, $\mathrm{NCH}_{2}$ ), 3.49 (s, $3 \mathrm{H}, \mathrm{OCH}_{3}$ ); MS (ESI) calcd for $\mathrm{C}_{22} \mathrm{H}_{22}-$ $\mathrm{NO}_{8}[\mathrm{M}+\mathrm{H}]^{+} 428.1345$, found 428.1340 .

7-甲氧甲氧基-4'-(哌啶- $N$-甲酰氧基)-5-羟基异黄酮 (5f): 类白色固体, 产率 $79.6 \%$. m.p. $161 \sim 162{ }^{\circ} \mathrm{C} ;{ }^{1} \mathrm{H}$ NMR $\left(\mathrm{CDCl}_{3}, 400 \mathrm{MHz}\right) \delta: 12.76(\mathrm{~s}, 1 \mathrm{H}, 5-\mathrm{OH}), 7.90$ (s, $1 \mathrm{H}, 2-\mathrm{H}), 7.52$ (d, $\left.J=8.4 \mathrm{~Hz}, 2 \mathrm{H}, 2^{\prime}, 6^{\prime}-\mathrm{H}\right), 7.19$ (d, $J=8.4$ $\left.\mathrm{Hz}, 2 \mathrm{H}, 3^{\prime}, 5^{\prime}-\mathrm{H}\right), 6.58$ (d, $\left.J=1.6 \mathrm{~Hz}, 1 \mathrm{H}, 6-\mathrm{H}\right), 6.50$ (d, $J=$ $1.6 \mathrm{~Hz}, 1 \mathrm{H}, 8-\mathrm{H}), 5.24\left(\mathrm{~s}, 2 \mathrm{H}, \mathrm{OCH}_{2} \mathrm{O}\right), 3.57 \sim 3.71(\mathrm{~m}$, $\left.4 \mathrm{H}, 2 \times \mathrm{NCH}_{2}\right), 3.50\left(\mathrm{~s}, 3 \mathrm{H}, \mathrm{OCH}_{3}\right), 1.65\left(\mathrm{~s}, 6 \mathrm{H}, 3 \times \mathrm{CH}_{2}\right)$; MS (ESI) calcd for $\mathrm{C}_{23} \mathrm{H}_{24} \mathrm{NO}_{7}[\mathrm{M}+\mathrm{H}]^{+} 426.1553$, found 426.1562 .

7-甲氧甲氧基-4'-(4-甲基哌溙- $N$-甲酰氧基)-5-羟基 异黄酮 (5g): 浅黄色固体, 产率 62.5\%. m.p. 189 190 
${ }^{\circ} \mathrm{C} ;{ }^{1} \mathrm{H}$ NMR $\left(\mathrm{CDCl}_{3}, 400 \mathrm{MHz}\right) \delta: 12.75$ (s, 1H, 5-OH), 7.90 (s, 1H, 2-H), 7.53 (d, $\left.J=8.8 \mathrm{~Hz}, 2 \mathrm{H}, 2^{\prime}, 66^{\prime}-\mathrm{H}\right), 7.19$ (d, $\left.J=8.8 \mathrm{~Hz}, 2 \mathrm{H}, 3^{\prime}, 5^{\prime}-\mathrm{H}\right), 6.58(\mathrm{~d}, J=2.0 \mathrm{~Hz}, 1 \mathrm{H}, 6-\mathrm{H}), 6.50$ (d, $J=2.0 \mathrm{~Hz}, 1 \mathrm{H}, 8-\mathrm{H}), 5.24\left(\mathrm{~s}, 2 \mathrm{H}, \mathrm{OCH}_{2} \mathrm{O}\right), 3.73(\mathrm{~s}, 2 \mathrm{H}$, $\mathrm{NCH}_{2}$ ), 3.65 (s, $2 \mathrm{H}, \mathrm{NCH}_{2}$ ), 3.50 (s, 3H, $\left.\mathrm{OCH}_{3}\right), 2.50$ (s, $4 \mathrm{H}, 2 \times \mathrm{NCH}_{2}$ ), 2.38 (s, $3 \mathrm{H}, \mathrm{NCH}_{3}$ ); MS (ESI) calcd for $\mathrm{C}_{23} \mathrm{H}_{25} \mathrm{~N}_{2} \mathrm{O}_{7}[\mathrm{M}+\mathrm{H}]^{+}$441.1662, found 441.1667.

7-甲氧甲氧基-4'-(4-芐基哌嗪- $N$-甲酰氧基)-5-羟基 异黄酮 $(5 \mathbf{h})$ : 类白色固体，产率 $85.4 \%$. m.p. 161 162 ${ }^{\circ} \mathrm{C} ;{ }^{1} \mathrm{H}$ NMR $\left(\mathrm{CDCl}_{3}, 400 \mathrm{MHz}\right) \delta: 12.75(\mathrm{~s}, 1 \mathrm{H}, 5-\mathrm{OH})$, $7.90(\mathrm{~s}, 1 \mathrm{H}, 2-\mathrm{H}), 7.52$ (d, $\left.J=8.4 \mathrm{~Hz}, 2 \mathrm{H}, 2^{\prime}, 6^{\prime}-\mathrm{H}\right), 7.28 \sim$ 7.35 (m, 5H, Ar-H), 7.19 (d, $\left.J=8.4 \mathrm{~Hz}, 2 \mathrm{H}, 3^{\prime}, 5^{\prime}-\mathrm{H}\right), 6.58$ $(\mathrm{d}, J=2.0 \mathrm{~Hz}, 1 \mathrm{H}, 6-\mathrm{H}), 6.50(\mathrm{~d}, J=2.0 \mathrm{~Hz}, 1 \mathrm{H}, 8-\mathrm{H})$, $5.24\left(\mathrm{~s}, 2 \mathrm{H}, \mathrm{OCH}_{2} \mathrm{O}\right), 3.70\left(\mathrm{~s}, 2 \mathrm{H}, \mathrm{NCH}_{2}\right), 3.58(\mathrm{~s}, 4 \mathrm{H}$, $\left.2 \times \mathrm{NCH}_{2}\right), 3.50\left(\mathrm{~s}, 3 \mathrm{H}, \mathrm{OCH}_{3}\right), 2.51\left(\mathrm{~s}, 4 \mathrm{H}, 2 \times \mathrm{NCH}_{2}\right)$; MS (ESI) calcd for $\mathrm{C}_{29} \mathrm{H}_{29} \mathrm{~N}_{2} \mathrm{O}_{7}[\mathrm{M}+\mathrm{H}]^{+}$517.1975, found 517.1968.

\subsection{4 '-氨基甲酰氧基-5,7-二羟基异黄酮(6a～6h)的制 备}

在反应瓶中加入 $\mathbf{5 a} \sim 5 \mathbf{h}(0.25 \mathrm{mmol})$ 、乙醇 $(5 \mathrm{~mL})$ 和质量分数为 $10 \% \mathrm{HCl}$ 水溶液 $(5 \mathrm{~mL})$, 升温回流摚拌反 应 $3 \mathrm{~h}$, 反应结束后, 减压蒸除溶剂, 粗品经硅胶柱层析 纯化 (流动相: $V$ (二氯甲烷) $: V$ (丙酮 $)=30 ： 1$ ), 得 4 '-氨 基甲酰氧基-5,7-二差基异黄酮 $(6 \mathbf{a} \sim 6 \mathbf{h})$, 其性状、产率、 熔点及波谱数据如下:

4'-( $N, N$-二乙氨甲酰氧基)-5,7-二羟基异黄酮 (6a): 浅黄色固体, 产率 $85.4 \%$. m.p. $182 \sim 184{ }^{\circ} \mathrm{C} ;{ }^{1} \mathrm{H}$ NMR (DMSO- $\left.d_{6}, 400 \mathrm{MHz}\right) \delta: 12.87$ (s, 1H, 5-OH), 10.95 (s, $1 \mathrm{H}, 7-\mathrm{OH}), 8.45$ (s, 1H, 2-H), 7.57 (d, $J=8.4 \mathrm{~Hz}, 2 \mathrm{H}$, 2',6'-H), 7.19 (d, $\left.J=8.4 \mathrm{~Hz}, 2 \mathrm{H}, 3^{\prime}, 5^{\prime}-\mathrm{H}\right), 6.42$ (d, $J=2.0$ Hz, 1H, 6-H), 6.25 (d, $J=2.0 \mathrm{~Hz}, 1 \mathrm{H}, 8-\mathrm{H}), 3.40$ (q, $J=$ $6.8 \mathrm{~Hz}, 2 \mathrm{H}, \mathrm{NCH}_{2}$ ), 3.33 (q, $J=6.8 \mathrm{~Hz}, 2 \mathrm{H}, \mathrm{NCH}_{2}$ ), 1.21 (t, $\left.J=6.8 \mathrm{~Hz}, 3 \mathrm{H}, \mathrm{CH}_{3}\right), 1.12\left(\mathrm{t}, J=6.8 \mathrm{~Hz}, 3 \mathrm{H}, \mathrm{CH}_{3}\right.$ ); HRMS (ESI) calcd for $\mathrm{C}_{20} \mathrm{H}_{20} \mathrm{NO}_{6}[\mathrm{M}+\mathrm{H}]^{+} 370.1291$, found 370.1288 .

4'-( $N, N$-二甲氨基甲酰氧基)-5,7-二羟基异黄酮 (6b): 类白色固体, 产率 85.0\%. m.p. $238 \sim 239{ }^{\circ} \mathrm{C} ;{ }^{1} \mathrm{H}$ NMR (DMSO- $\left.d_{6}, 400 \mathrm{MHz}\right) \delta: 12.88(\mathrm{~s}, 1 \mathrm{H}, 5-\mathrm{OH}), 10.96(\mathrm{~s}$, $1 \mathrm{H}, 7-\mathrm{OH}), 8.46$ (s, 1H, 2-H), 7.57 (d, $J=8.4 \mathrm{~Hz}, 2 \mathrm{H}, 2^{\prime}$, 6'-H), 7.19 (d, $\left.J=8.4 \mathrm{~Hz}, 2 \mathrm{H}, 3^{\prime}, 5^{\prime}-\mathrm{H}\right), 6.42$ (d, $J=2.0 \mathrm{~Hz}$, $1 \mathrm{H}, 6-\mathrm{H}), 6.25$ (d, $J=2.0 \mathrm{~Hz}, 1 \mathrm{H}, 8-\mathrm{H}), 3.06$ (s, $3 \mathrm{H}$, $\mathrm{NCH}_{3}$ ), 2.93 (s, 3H, $\mathrm{NCH}_{3}$ ); HRMS (ESI) calcd for $\mathrm{C}_{18} \mathrm{H}_{16} \mathrm{NO}_{6}[\mathrm{M}+\mathrm{H}]^{+}$342.0978, found 342.0976.

4'-( $N$-甲基- $N$-乙基氨甲酰氧基)-5,7-二羟基异黄酮 (6c): 浅黄色固体, 产率 $85.5 \%$. m.p. $183 \sim 185{ }^{\circ} \mathrm{C} ;{ }^{1} \mathrm{H}$
NMR (DMSO- $\left.d_{6}, 400 \mathrm{MHz}\right) \delta: 12.87$ (s, $\left.1 \mathrm{H}, 5-\mathrm{OH}\right), 10.95$ (s, 1H, 7-OH), 8.45 (s, 1H, 2-H), 7.57 (d, $J=8.4 \mathrm{~Hz}, 2 \mathrm{H}$, 2',6'-H), 7.19 (d, $\left.J=8.4 \mathrm{~Hz}, 2 \mathrm{H}, 3^{\prime}, 5^{\prime}-\mathrm{H}\right), 6.42$ (d, $J=2.0$ $\mathrm{Hz}, 1 \mathrm{H}, 6-\mathrm{H}), 6.25$ (d, $J=2.0 \mathrm{~Hz}, 1 \mathrm{H}, 8-\mathrm{H}), 3.30 \sim 3.45$ $\left(\mathrm{m}, 2 \mathrm{H}, \mathrm{NCH}_{2}\right), 3.04\left(\mathrm{~s}, 1.5 \mathrm{H}, 1 / 2 \mathrm{NCH}_{3}\right), 2.91(\mathrm{~s}, 1.5 \mathrm{H}$, $\left.1 / 2 \mathrm{NCH}_{3}\right), 1.09 \sim 1.21\left(\mathrm{~m}, 3 \mathrm{H}, \mathrm{CH}_{3}\right)$; HRMS (ESI) calcd for $\mathrm{C}_{19} \mathrm{H}_{18} \mathrm{NO}_{6}[\mathrm{M}+\mathrm{H}]^{+}$356.1134, found 356.1137.

4'-( $N, N$-二异丙胺甲酰氧基)-5,7-二羟基异黄酩 (6d): 浅灰色固体, 产率 $86.0 \%$. m.p. $230 \sim 231{ }^{\circ} \mathrm{C} ;{ }^{1} \mathrm{H}$ NMR (DMSO- $\left.d_{6}, 400 \mathrm{MHz}\right) \delta: 12.88$ (s, 1H, 5-OH), 10.95 (s, $1 \mathrm{H}, 7-\mathrm{OH}), 8.45$ (s, 1H, 2-H), 7.57 (d, $J=8.4 \mathrm{~Hz}, 2 \mathrm{H}$, 2',6'-H), 7.17 (d, $\left.J=8.4 \mathrm{~Hz}, 2 \mathrm{H}, 3^{\prime}, 5^{\prime}-\mathrm{H}\right), 6.42$ (d, $J=2.0$ $\mathrm{Hz}, 1 \mathrm{H}, 6-\mathrm{H}), 6.25$ (d, $J=2.0 \mathrm{~Hz}, 1 \mathrm{H}, 8-\mathrm{H}), 4.00$ (brs, $2 \mathrm{H}$, $2 \times \mathrm{NCH}$ ), 1.27 (brs, $12 \mathrm{H}, 4 \times \mathrm{CH}_{3}$ ); HRMS (ESI) calcd for $\mathrm{C}_{22} \mathrm{H}_{24} \mathrm{NO}_{6}[\mathrm{M}+\mathrm{H}]^{+}$398.1604, found 398.1597.

4'-(吗啡啉- $N$-甲酰氧基)-5,7-二羟基异黄酮(6e): 类 白色固体, 产率 $65.0 \%$. m.p. $243 \sim 244{ }^{\circ} \mathrm{C} ;{ }^{1} \mathrm{H}$ NMR (DMSO- $\left.d_{6}, 400 \mathrm{MHz}\right) \delta: 12.87(\mathrm{~s}, 1 \mathrm{H}, 5-\mathrm{OH}), 10.96$ (s, $1 \mathrm{H}, 7-\mathrm{OH}), 8.46(\mathrm{~s}, 1 \mathrm{H}, 2-\mathrm{H}), 7.58(\mathrm{~d}, J=8.8 \mathrm{~Hz}, 2 \mathrm{H}$, $\left.2^{\prime}, 6^{\prime}-\mathrm{H}\right), 7.21$ (d, $\left.J=8.4 \mathrm{~Hz}, 2 \mathrm{H}, 3^{\prime}, 5^{\prime}-\mathrm{H}\right), 6.42$ (d, $J=2.0$ $\mathrm{Hz}, 1 \mathrm{H}, 6-\mathrm{H}), 6.25$ (d, $J=2.0 \mathrm{~Hz}, 1 \mathrm{H}, 8-\mathrm{H}), 3.60 \sim 3.66$ (m, $4 \mathrm{H}, 2 \times \mathrm{OCH}_{2}$ ), 3.43 (brs, $2 \mathrm{H}, \mathrm{NCH}_{2}$ ), 3.35 (brs, $2 \mathrm{H}$, $\mathrm{NCH}_{2}$ ); HRMS (ESI) calcd for $\mathrm{C}_{20} \mathrm{H}_{18} \mathrm{NO}_{7}[\mathrm{M}+\mathrm{H}]^{+}$ 384.1083 , found 384.1081 .

4'-(哌啶- $N$-甲酰氧基)-5,7-二羟基异黄酮(6f): 类白 色固体，产率 84.2\%. m.p. $216 \sim 217{ }^{\circ} \mathrm{C} ;{ }^{1} \mathrm{H}$ NMR (DMSO- $\left.d_{6}, 400 \mathrm{MHz}\right) \delta: 12.87(\mathrm{~s}, 1 \mathrm{H}, 5-\mathrm{OH}), 10.95(\mathrm{~s}$, $1 \mathrm{H}, 7-\mathrm{OH}), 8.46(\mathrm{~s}, 1 \mathrm{H}, 2-\mathrm{H}), 7.57$ (d, $J=8.8 \mathrm{~Hz}, 2 \mathrm{H}$, 2',6'-H), 7.18 (d, $\left.J=8.8 \mathrm{~Hz}, 2 \mathrm{H}, 3^{\prime}, 5^{\prime}-\mathrm{H}\right), 6.42$ (d, $J=2.0$ $\mathrm{Hz}, 1 \mathrm{H}, 6-\mathrm{H}), 6.25$ (d, $J=2.0 \mathrm{~Hz}, 1 \mathrm{H}, 8-\mathrm{H}), 3.56$ (brs, $2 \mathrm{H}$, $\left.\mathrm{NCH}_{2}\right), 3.41$ (brs, $\left.2 \mathrm{H}, \mathrm{NCH}_{2}\right), 1.56 \sim 1.59(\mathrm{~m}, 6 \mathrm{H}$, $3 \times \mathrm{CH}_{2}$ ); HRMS (ESI) calcd for $\mathrm{C}_{21} \mathrm{H}_{20} \mathrm{NO}_{6}[\mathrm{M}+\mathrm{H}]^{+}$ 382.1291 , found 382.1283 .

4'-(4-甲基哌嗪- $\mathrm{N}$-甲酰氧基)-5,7-二羊基异黄酮盐酸 盐 $(6 \mathbf{g})$ : 类白色固体, 产率 $67.0 \%$. m.p. $>260{ }^{\circ} \mathrm{C} ;{ }^{1} \mathrm{H}$ NMR (DMSO- $\left.d_{6}, 400 \mathrm{MHz}\right) \delta: 12.85$ (s, $\left.1 \mathrm{H}, 5-\mathrm{OH}\right), 11.10$ (brs, $\left.1 \mathrm{H}, \mathrm{NH}^{+}\right), 11.06(\mathrm{~s}, 1 \mathrm{H}, 7-\mathrm{OH}), 8.47(\mathrm{~s}, 1 \mathrm{H}, 2-\mathrm{H})$, $7.60\left(\mathrm{~d}, J=8.4 \mathrm{~Hz}, 2 \mathrm{H}, 2^{\prime}, 6{ }^{\prime}-\mathrm{H}\right), 7.24(\mathrm{~d}, J=8.4 \mathrm{~Hz}, 2 \mathrm{H}$, 3', 5'-H), 6.45 (d, $J=2.0 \mathrm{~Hz}, 1 \mathrm{H}, 6-\mathrm{H}), 6.27$ (d, $J=2.0 \mathrm{~Hz}$, $1 \mathrm{H}, 8-\mathrm{H}), 4.28 \sim 4.13\left(\mathrm{~m}, 2 \mathrm{H}, \mathrm{NCH}_{2}\right), 3.44 \sim 3.14(\mathrm{~m}, 6 \mathrm{H}$, $\left.3 \times \mathrm{NCH}_{2}\right), 2.80\left(\mathrm{~s}, 3 \mathrm{H}, \mathrm{NCH}_{3}\right)$; HRMS (ESI) calcd for $\mathrm{C}_{21} \mathrm{H}_{21} \mathrm{~N}_{2} \mathrm{O}_{6}[\mathrm{M}-\mathrm{Cl}]^{+}$397.1400, found 397.1406.

4'-(4-苄基哌嗪- $\mathrm{N}$-甲酰氧基)-5,7-二美基异黄酮盐酸 盐 $(\mathbf{6 h})$ : 类白色固体, 产率 $81.2 \%$. m.p. $>260{ }^{\circ} \mathrm{C} ;{ }^{1} \mathrm{H}$ NMR (DMSO- $\left.d_{6}, 400 \mathrm{MHz}\right) \delta$ : 12.85 (s, $\left.1 \mathrm{H}, 5-\mathrm{OH}\right), 11.39$ 
(s, 1H, $\left.\mathrm{NH}^{+}\right), 11.05(\mathrm{~s}, 1 \mathrm{H}, 7-\mathrm{OH}), 8.47(\mathrm{~s}, 1 \mathrm{H}, 2-\mathrm{H})$, $7.64 \sim 7.63(\mathrm{~m}, 2 \mathrm{H}, \operatorname{Ar}-\mathrm{H}), 7.60(\mathrm{~d}, J=8.4 \mathrm{~Hz}, 2 \mathrm{H}$, $\left.2^{\prime}, 6^{\prime}-\mathrm{H}\right), 7.49 \sim 7.47(\mathrm{~m}, 3 \mathrm{H}, \mathrm{Ar}-\mathrm{H}), 7.23(\mathrm{~d}, J=8.4 \mathrm{~Hz}$, $\left.2 \mathrm{H}, 3^{\prime}, 5^{\prime}-\mathrm{H}\right), 6.45$ (d, $\left.J=2.0 \mathrm{~Hz}, 1 \mathrm{H}, 6-\mathrm{H}\right), 6.27$ (d, $J=2.0$ $\mathrm{Hz}, 1 \mathrm{H}, 8-\mathrm{H}), 4.38 \sim 4.12\left(\mathrm{~m}, 4 \mathrm{H}, 2 \times \mathrm{NCH}_{2}\right), 3.60 \sim 3.35$ (m, $4 \mathrm{H}, 2 \times \mathrm{NCH}_{2}$ ), 3.17 (brs, $2 \mathrm{H}, \mathrm{NCH}_{2}$ ); HRMS (ESI) calcd for $\mathrm{C}_{27} \mathrm{H}_{25} \mathrm{~N}_{2} \mathrm{O}_{6}[\mathrm{M}-\mathrm{Cl}]^{+}$473.1713, found 473.1715 .

\subsection{7-氨基甲酰氧基-5,4'-二羟基异黄酮( $7 \mathrm{a} \sim 7 \mathrm{~h}$ )的制 备}

在反应瓶中加入染料木素 $\mathbf{1}(0.15 \mathrm{~g}, 0.56 \mathrm{mmol})$, $\mathrm{KOH}(0.032 \mathrm{~g}, 0.56 \mathrm{mmol})$ 和 $N, N$-二甲基甲酰胺 $(10 \mathrm{~mL})$, 室温搅拌反应 $1 \mathrm{~h}$ 后加入相应的氨基甲酰氯 $(\mathbf{3 a} \sim \mathbf{3 h})$ $(0.71 \mathrm{mmol})$, 继续室温搅拌反应 $12 \sim 15 \mathrm{~h}$, 反应结束后, 将反应液倒入去离子水 $(60 \mathrm{~mL})$ 中, 用 $10 \% \mathrm{HCl}$ 水溶液 调节溶液 $\mathrm{pH}$ 至酸性, 过滤析出的类白色固体, 所得粗 品经硅胶柱层析纯化 [流动相: $V$ (二氯甲烷 $): V$ (丙酮 $)=$ $30: 1$ ], 得 7-氨基甲酰氧基-5,4'-二羟基异黄酮(7a 7h). 其性状、产率、熔点及波谱数据如下.

7-( $N, N$-二乙氨甲酰氧基)-5,4'-二羟基异黄酮 (7a): 浅黄色固体, 产率 $49.0 \%$. m.p. $196 \sim 198{ }^{\circ} \mathrm{C} ;{ }^{1} \mathrm{H}$ NMR (DMSO- $\left.d_{6}, 400 \mathrm{MHz}\right) \delta: 12.93$ (s, 1H, 5-OH), 9.65 (s, $1 \mathrm{H}$, 4'-OH), 8.50 (s, 1H, 2-H), 7.41 (d, $\left.J=8.4 \mathrm{~Hz}, 2 \mathrm{H}, 2^{\prime}, 66^{\prime}-\mathrm{H}\right)$, $6.97(\mathrm{~d}, J=2.0 \mathrm{~Hz}, 1 \mathrm{H}, 6-\mathrm{H}), 6.84(\mathrm{~d}, J=8.4 \mathrm{~Hz}, 2 \mathrm{H}$, $\left.3^{\prime}, 5^{\prime}-\mathrm{H}\right), 6.65$ (d, $\left.J=2.0 \mathrm{~Hz}, 1 \mathrm{H}, 8-\mathrm{H}\right), 3.41$ (q, $J=6.8 \mathrm{~Hz}$, $2 \mathrm{H}, \mathrm{NCH}_{2}$ ), 3.32 (q, $\left.J=6.8 \mathrm{~Hz}, 2 \mathrm{H}, \mathrm{NCH}_{2}\right), 1.20$ (t, $J=6.8$ $\mathrm{Hz}, 3 \mathrm{H}, \mathrm{CH}_{3}$ ), 1.13 (t, $J=6.8 \mathrm{~Hz}, 3 \mathrm{H}, \mathrm{CH}_{3}$ ); HRMS (ESI) calcd for $\mathrm{C}_{20} \mathrm{H}_{20} \mathrm{NO}_{6}[\mathrm{M}+\mathrm{H}]^{+}$370.1291, found 370.1296.

7-( $N, N$-二甲氨甲酰氧基)-5,4'-二羟基异黄酮 (7b): 浅黄色固体, 产率 $45.0 \%$. m.p. $>260{ }^{\circ} \mathrm{C} ;{ }^{1} \mathrm{H}$ NMR (DMSO- $\left.d_{6}, 400 \mathrm{MHz}\right) \delta: 12.93$ (s, $\left.1 \mathrm{H}, 5-\mathrm{OH}\right), 9.65(\mathrm{~s}, 1 \mathrm{H}$, 4'-OH), 8.50 (s, 1H, 2-H), 7.41 (d, $\left.J=8.4 \mathrm{~Hz}, 2 \mathrm{H}, 2^{\prime}, 6^{\prime}-\mathrm{H}\right)$, 6.97 (d, $J=1.6 \mathrm{~Hz}, 1 \mathrm{H}, 6-\mathrm{H}), 6.84(\mathrm{~d}, J=8.4 \mathrm{~Hz}, 2 \mathrm{H}$, 3',5'-H), 6.65 (d, $J=1.6 \mathrm{~Hz}, 1 \mathrm{H}, 8-\mathrm{H}), 3.05\left(\mathrm{~s}, 3 \mathrm{H}, \mathrm{NCH}_{3}\right.$ ), $2.93\left(\mathrm{~s}, 3 \mathrm{H}, \mathrm{NCH}_{3}\right.$ ); HRMS (ESI) calcd for $\mathrm{C}_{18} \mathrm{H}_{16} \mathrm{NO}_{6}$ $[\mathrm{M}+\mathrm{H}]^{+}$342.0978, found 342.0972 .

7-( $N$-甲基- $N$-乙基氨甲酰氧基)-5,4'-二羟基异黄酮 (7c): 浅黄色固体, 产率 $40.0 \%$. m.p. $230 \sim 232{ }^{\circ} \mathrm{C} ;{ }^{1} \mathrm{H}$ NMR (DMSO- $\left.d_{6}, 400 \mathrm{MHz}\right) \delta: 12.93$ (s, 1H, 5-OH), 9.66 (s, 1H, 4'-OH), 8.50 (s, 1H, 2-H), 7.41 (d, $J=8.4 \mathrm{~Hz}, 2 \mathrm{H}$, 2', 6'-H), 6.97 (d, $J=2.0 \mathrm{~Hz}, 1 \mathrm{H}, 6-\mathrm{H}), 6.84(\mathrm{~d}, J=8.4 \mathrm{~Hz}$, $\left.2 \mathrm{H}, 3^{\prime}, 5^{\prime}-\mathrm{H}\right), 6.65(\mathrm{~d}, J=2.0 \mathrm{~Hz}, 1 \mathrm{H}, 8-\mathrm{H}), 3.45 \sim 3.30(\mathrm{~m}$, $\left.2 \mathrm{H}, \mathrm{NCH}_{2}\right), 3.03\left(\mathrm{~s}, 1.5 \mathrm{H}, 1 / 2 \mathrm{NCH}_{3}\right), 2.92(\mathrm{~s}, 1.5 \mathrm{H}$, $1 / 2 \mathrm{NCH}_{3}$ ), $1.20 \sim 1.10\left(\mathrm{~m}, 3 \mathrm{H}, \mathrm{CH}_{3}\right.$ ); HRMS (ESI) calcd for $\mathrm{C}_{19} \mathrm{H}_{18} \mathrm{NO}_{6}[\mathrm{M}+\mathrm{H}]^{+}$356.1134, found 356.1126.
7-(N,N-二异丙胺甲酰氧基)-5,4'-二垟基异黄酤(7d): 类白色固体, 产率 $40.8 \%$. m.p. $224 \sim 225{ }^{\circ} \mathrm{C} ;{ }^{1} \mathrm{H}$ NMR (DMSO- $\left.d_{6}, 400 \mathrm{MHz}\right) \delta: 12.94$ (s, 1H, 5-OH), $9.65(\mathrm{~s}, 1 \mathrm{H}$, 4'-OH), 8.50 (s, 1H, 2-H), 7.41 (d, J=8.8 Hz, 2H, 2',6'-H), $6.94(\mathrm{~d}, J=2.0 \mathrm{~Hz}, 1 \mathrm{H}, 6-\mathrm{H}), 6.84(\mathrm{~d}, J=8.8 \mathrm{~Hz}, 2 \mathrm{H}$, 3',5'-H), 6.62 (d, $J=2.0 \mathrm{~Hz}, 1 \mathrm{H}, 8-\mathrm{H}), 4.00$ (brs, $2 \mathrm{H}$, $2 \times \mathrm{NCH}$ ), 1.26 (brs, $12 \mathrm{H}, 4 \times \mathrm{CH}_{3}$ ); HRMS (ESI) calcd for $\mathrm{C}_{22} \mathrm{H}_{24} \mathrm{NO}_{6}[\mathrm{M}+\mathrm{H}]^{+}$398.1604, found 398.1596.

7-(吗啡啉- $N$-甲酰氧基)-5,4'-二羟基异黄酥(7e): 浅 黄色固体, 产率 $48.0 \%$. m.p. $221 \sim 223{ }^{\circ} \mathrm{C} ;{ }^{1} \mathrm{H}$ NMR (DMSO- $\left.d_{6}, 400 \mathrm{MHz}\right) \delta: 12.94$ (s, 1H, 5-OH), $9.65(\mathrm{~s}, 1 \mathrm{H}$, 4'-OH), 8.50 (s, 1H, 2-H), 7.41 (d $\left.J=8.4 \mathrm{~Hz}, 2 \mathrm{H}, 2^{\prime}, 66^{\prime}-\mathrm{H}\right)$, $7.01(\mathrm{~d}, J=2.0 \mathrm{~Hz}, 1 \mathrm{H}, 6-\mathrm{H}), 6.84(\mathrm{~d}, J=8.4 \mathrm{~Hz}, 2 \mathrm{H}$, $\left.3^{\prime}, 5^{\prime}-\mathrm{H}\right), 6.70(\mathrm{~d}, J=2.0 \mathrm{~Hz}, 1 \mathrm{H}, 8-\mathrm{H}), 3.67 \sim 3.65(\mathrm{~m}, 4 \mathrm{H}$, $2 \times \mathrm{OCH}_{2}$ ), 3.59 (brs, $2 \mathrm{H}, \mathrm{NCH}_{2}$ ), 3.44 (brs, $2 \mathrm{H}, \mathrm{NCH}_{2}$ ); HRMS (ESI) calcd for $\mathrm{C}_{20} \mathrm{H}_{18} \mathrm{NO}_{7}[\mathrm{M}+\mathrm{H}]^{+} 384.1083$, found 384.1078 .

7-(哌啶- $N$-甲酰氧基)-5,4'-二羟基异黄酮(7f): 浅黄 色色固体, 产率 $48.0 \%$. m.p. $232 \sim 233{ }^{\circ} \mathrm{C} ;{ }^{1} \mathrm{H}$ NMR (DMSO- $\left.d_{6}, 400 \mathrm{MHz}\right) \delta: 12.93(\mathrm{~s}, 1 \mathrm{H}, 5-\mathrm{OH}), 9.65(\mathrm{~s}, 1 \mathrm{H}$, 4'-OH), 8.50 (s, 1H, 2-H), 7.41 (d, J=8.4 Hz, 2H, 2',6'-H), $6.97(\mathrm{~d}, J=2.0 \mathrm{~Hz}, 1 \mathrm{H}, 6-\mathrm{H}), 6.84(\mathrm{~d}, J=8.4 \mathrm{~Hz}, 2 \mathrm{H}$, 3',5'-H), 6.66 (d, $J=2.0 \mathrm{~Hz}, 1 \mathrm{H}, 8-\mathrm{H}), 3.55$ (s, $2 \mathrm{H}, \mathrm{NCH}_{2}$ ), $3.42\left(\mathrm{~s}, 2 \mathrm{H}, \mathrm{NCH}_{2}\right), 1.59$ (brs, $6 \mathrm{H}, 3 \times \mathrm{CH}_{2}$ ); HRMS (ESI) calcd for $\mathrm{C}_{21} \mathrm{H}_{20} \mathrm{NO}_{6}[\mathrm{M}+\mathrm{H}]^{+}$382.1291, found 382.1288 .

7-(4-甲基哌嗪- $N$-甲酰氧基)-5,4'-二羟基异黄酮(7g): 浅黄色色固体，产率 47.8\%. m.p. 207 209 ${ }^{\circ} \mathrm{C} ;{ }^{1} \mathrm{H}$ NMR (DMSO-d $\left.d_{6}, 400 \mathrm{MHz}\right) \delta: 12.93$ (s, 1H, 5-OH), 9.65 (s, 1H, 4'-OH), 8.50 (s, 1H, 2-H), 7.40 (d, $\left.J=8.4 \mathrm{~Hz}, 2 \mathrm{H}, 2^{\prime}, 66^{\prime}-\mathrm{H}\right)$, 6.99 (d, $J=1.6 \mathrm{~Hz}, 1 \mathrm{H}, 6-\mathrm{H}), 6.84(\mathrm{~d}, J=8.4 \mathrm{~Hz}, 2 \mathrm{H}$, 3',5'-H), 6.67 (d, $J=1.6 \mathrm{~Hz}, 1 \mathrm{H}, 8-\mathrm{H}), 3.59$ (brs, $2 \mathrm{H}$, $\mathrm{NCH}_{2}$ ), 3.45 (brs, $2 \mathrm{H}, \mathrm{NCH}_{2}$ ), 2.40 (brs, $4 \mathrm{H}, 2 \times \mathrm{NCH}_{2}$ ), 2.25 (s, 3H, $\mathrm{NCH}_{3}$ ); HRMS (ESI) calcd for $\mathrm{C}_{21} \mathrm{H}_{21} \mathrm{~N}_{2} \mathrm{O}_{6}$ $[\mathrm{M}-\mathrm{Cl}]^{+}$397.1400, found 397.1398.

7-(4-芐基哌嗪- $N$-甲酰氧基)-5,4'-二羟基异黄酮(7h): 浅黄色色固体, 产率 43.1\%. m.p. 178 180 ${ }^{\circ} \mathrm{C} ;{ }^{1} \mathrm{H}$ NMR (DMSO- $\left.d_{6}, 400 \mathrm{MHz}\right) \delta: 12.93(\mathrm{~s}, 1 \mathrm{H}, 5-\mathrm{OH}), 9.65(\mathrm{~s}, 1 \mathrm{H}$, 4'-OH), 8.50 (s, 1H, 2-H), 7.40 (d $\left.J=8.4 \mathrm{~Hz}, 2 \mathrm{H}, 2^{\prime}, 6^{\prime}-\mathrm{H}\right)$, $7.37 \sim 7.27$ (m, 5H, Ar-H), $6.98(\mathrm{~d}, J=1.6 \mathrm{~Hz}, 1 \mathrm{H}, 6-\mathrm{H})$, $6.84\left(\mathrm{~d}, J=8.4 \mathrm{~Hz}, 2 \mathrm{H}, 3^{\prime}, 5^{\prime}-\mathrm{H}\right), 6.67$ (d, $J=1.6 \mathrm{~Hz}, 1 \mathrm{H}$, 8-H), 3.59 (brs, $2 \mathrm{H}, \mathrm{NCH}_{2}$ ), 3.53 (s, $2 \mathrm{H}, \mathrm{NCH}_{2}$ ), 3.45 (brs, $2 \mathrm{H}, \mathrm{NCH}_{2}$ ), 2.44 (brs, $4 \mathrm{H}, 2 \times \mathrm{NCH}_{2}$ ); HRMS (ESI) calcd for $\mathrm{C}_{27} \mathrm{H}_{25} \mathrm{~N}_{2} \mathrm{O}_{6}[\mathrm{M}-\mathrm{Cl}]^{+}$473.1713, found 473.1720. 


\subsection{7,4'-二(氨基甲酰氧基)-5-羟基异黄酮(8a 8h)的 制备}

在反应瓶中加入染料木素 $\mathbf{1}(0.15 \mathrm{~g}, 0.56 \mathrm{mmol})$ 、无 水碳酸钾 $(0.42 \mathrm{~g}, 3.05 \mathrm{mmol})$ 、丙酮 $(10 \mathrm{~mL})$ 和相应的氨基 甲酰氯 $(\mathbf{3 a} \sim 2 \mathbf{h})(1.4 \mathrm{mmol})$, 升温回流搅拌反应 $12 \sim 15$ $\mathrm{h}$, 反应结束后, 减压蒸除溶剂, 残余物中加入去离子水 $(20 \mathrm{~mL})$, 用乙醚 $(25 \mathrm{~mL} \times 3)$ 萃取, 有机层合并后依次用 质量分数为 $10 \% \mathrm{NaOH}$ 水溶液 $(10 \mathrm{~mL} \times 2)$ 、去离子水 $(20$ $\mathrm{mL})$ 和饱和氯化钠水溶液 $(20 \mathrm{~mL})$ 洗涤, 经无水硫酸钠干 燥后过滤, 滤液减压蒸除溶剂, 粗品经硅胶柱层析纯化 [流动相: $V$ (二氯甲烷) $: V($ 丙酮 $)=30 ： 1$ ], 得 7,4'二(氨 基甲酰氧基)-5-羊基异黄酮( $8 \mathbf{a} \sim 8 \mathrm{~h})$, 其性状、产率、熔 点及波谱数据如下.

7,4'-二( $N, N$-二乙氨甲酰氧基)-5-羟基异黄酮 $(\mathbf{8 a})$ : 浅黄色固体, 产率 $61.5 \%$. m.p. $114 \sim 116{ }^{\circ} \mathrm{C} ;{ }^{1} \mathrm{H}$ NMR $\left(\mathrm{CDCl}_{3}, 400 \mathrm{MHz}\right) \delta: 12.74(\mathrm{~s}, 1 \mathrm{H}, 5-\mathrm{OH}), 7.94(\mathrm{~s}, 1 \mathrm{H}$, 2-H), 7.53 (d, $\left.J=8.4 \mathrm{~Hz}, 2 \mathrm{H}, 2^{\prime}, 6^{\prime}-\mathrm{H}\right), 7.21$ (d, $J=8.4 \mathrm{~Hz}$, $\left.2 \mathrm{H}, 3^{\prime}, 5^{\prime}-\mathrm{H}\right), 6.83$ (d, $\left.J=2.0 \mathrm{~Hz}, 1 \mathrm{H}, 6-\mathrm{H}\right), 6.61$ (d, $J=2.0$ $\mathrm{Hz}, 1 \mathrm{H}, 8-\mathrm{H}), 3.46 \sim 3.38\left(\mathrm{~m}, 8 \mathrm{H}, 4 \times \mathrm{NCH}_{2}\right), 1.28 \sim 1.22$ (m, $12 \mathrm{H}, 4 \times \mathrm{NCH}_{3}$ ); HRMS (ESI) calcd for $\mathrm{C}_{25} \mathrm{H}_{29} \mathrm{~N}_{2} \mathrm{O}_{7}$ $[\mathrm{M}+\mathrm{H}]^{+}$469.1975, found 469.1972.

$7,4^{\prime}$-二( $N, N$-二甲氨甲酰氧基)-5-羟基异黄酮 $(\mathbf{8 b})$ : 类白色固体, 产率 $60.0 \%$. m.p. 202 203 ${ }^{\circ} \mathrm{C} ;{ }^{1} \mathrm{H}$ NMR $\left(\mathrm{CDCl}_{3}, 400 \mathrm{MHz}\right) \delta: 12.74(\mathrm{~s}, 1 \mathrm{H}, 5-\mathrm{OH}), 7.95(\mathrm{~s}, 1 \mathrm{H}$, 2-H), 7.53 (d, $\left.J=8.8 \mathrm{~Hz}, 2 \mathrm{H}, 2^{\prime}, 6^{\prime}-\mathrm{H}\right), 7.21$ (d, $J=8.8 \mathrm{~Hz}$, $\left.2 \mathrm{H}, 3^{\prime}, 5^{\prime}-\mathrm{H}\right), 6.82(\mathrm{~d}, J=2.0 \mathrm{~Hz}, 1 \mathrm{H}, 6-\mathrm{H}), 6.61$ (d, $J=2.0$ $\mathrm{Hz}, 1 \mathrm{H}, 8-\mathrm{H}), 3.11\left(\mathrm{~s}, 6 \mathrm{H}, 2 \times \mathrm{NCH}_{3}\right), 3.04$ (s, 6H, $2 \times \mathrm{NCH}_{3}$ ); HRMS (ESI) calcd for $\mathrm{C}_{21} \mathrm{H}_{21} \mathrm{~N}_{2} \mathrm{O}_{7}[\mathrm{M}+\mathrm{H}]^{+}$ 413.1349, found 413.1353 .

7,4'-二 ( $N$-甲基- $N$-乙基氨甲酰氧基)- 5 -羟基异黄酮 (8c): 浅黄色固体, 产率 $65.0 \%$. m.p. $165 \sim 166{ }^{\circ} \mathrm{C} ;{ }^{1} \mathrm{H}$ NMR $\left(\mathrm{CDCl}_{3}, 400 \mathrm{MHz}\right) \delta: 12.75(\mathrm{~s}, 1 \mathrm{H}, 5-\mathrm{OH}), 7.95(\mathrm{~s}$, 1H, 2-H), 7.53 (d, $J=8.4 \mathrm{~Hz}, 2 \mathrm{H}, 2^{\prime}, 66^{\prime}-\mathrm{H}$ ), 7.21 (d, $J=8.4$ $\left.\mathrm{Hz}, 2 \mathrm{H}, 3^{\prime}, 5^{\prime}-\mathrm{H}\right), 6.83$ (d, $\left.J=1.6 \mathrm{~Hz}, 1 \mathrm{H}, 6-\mathrm{H}\right), 6.62$ (d, $J=$ $1.6 \mathrm{~Hz}, 1 \mathrm{H}, 8-\mathrm{H}), 3.52 \sim 3.40\left(\mathrm{~m}, 4 \mathrm{H}, 2 \times \mathrm{NCH}_{2}\right), 3.09(\mathrm{~d}$, $\left.J=4.4 \mathrm{~Hz}, 3 \mathrm{H}, \mathrm{NCH}_{3}\right), 3.01\left(\mathrm{~d}, J=4.4 \mathrm{~Hz}, 3 \mathrm{H}, \mathrm{NCH}_{3}\right)$, $1.27 \sim 1.19\left(\mathrm{~m}, 6 \mathrm{H}, 2 \times \mathrm{CH}_{3}\right)$; HRMS (ESI) calcd for $\mathrm{C}_{23} \mathrm{H}_{25} \mathrm{~N}_{2} \mathrm{O}_{7}[\mathrm{M}+\mathrm{H}]^{+}$441.1662, found 441.1655.

7,4'-二( $N, N$-二异丙胺甲酰氧基)-5-羟基异黄酮 $(\mathbf{8 d})$ : 类白色固体, 产率 $53.8 \%$. m.p. $150 \sim 151{ }^{\circ} \mathrm{C} ;{ }^{1} \mathrm{H}$ NMR $\left(\mathrm{CDCl}_{3}, 400 \mathrm{MHz}\right) \delta: 12.75(\mathrm{~s}, 1 \mathrm{H}, 5-\mathrm{OH}), 7.94(\mathrm{~s}, 1 \mathrm{H}$, 2-H), 7.53 (d, $\left.J=8.4 \mathrm{~Hz}, 2 \mathrm{H}, 2^{\prime}, 6^{\prime}-\mathrm{H}\right), 7.21$ (d, $J=8.4 \mathrm{~Hz}$, $\left.2 \mathrm{H}, 3^{\prime}, 5^{\prime}-\mathrm{H}\right), 6.82(\mathrm{~d}, J=2.0 \mathrm{~Hz}, 1 \mathrm{H}, 6-\mathrm{H}), 6.61$ (d, $J=2.0$ $\mathrm{Hz}, 1 \mathrm{H}, 8-\mathrm{H}$ ), 4.12 (brs, 2H, $2 \times \mathrm{NCH}$ ), 3.94 (brs, 2H, $2 \times \mathrm{NCH}$ ), 1.32 (brs, $24 \mathrm{H}, 8 \times \mathrm{CH}_{3}$ ); HRMS (ESI) calcd for $\mathrm{C}_{29} \mathrm{H}_{37} \mathrm{~N}_{2} \mathrm{O}_{7}[\mathrm{M}+\mathrm{H}]^{+}$525.2601, found 525.2607.

$7,4^{\prime}$-二(吗啡啉- $N$-甲酰氧基)-5-差基异黄酮 $(\mathbf{8 e})$ : 类 白色固体，产率 $65.0 \%$. m.p. $228 \sim 229{ }^{\circ} \mathrm{C} ;{ }^{1} \mathrm{H} \mathrm{NMR}$ $\left(\mathrm{CDCl}_{3}, 400 \mathrm{MHz}\right) \delta: 12.75(\mathrm{~s}, 1 \mathrm{H}, 5-\mathrm{OH}), 7.96(\mathrm{~s}, 1 \mathrm{H}$, 2-H), 7.53 (d, $\left.J=8.4 \mathrm{~Hz}, 2 \mathrm{H}, 2^{\prime}, 6^{\prime}-\mathrm{H}\right), 7.21$ (d, $J=8.4 \mathrm{~Hz}$, $\left.2 \mathrm{H}, 3^{\prime}, 5^{\prime}-\mathrm{H}\right), 6.82$ (d, $\left.J=2.0 \mathrm{~Hz}, 1 \mathrm{H}, 6-\mathrm{H}\right), 6.61$ (d, $J=2.0$ $\mathrm{Hz}, 1 \mathrm{H}, 8-\mathrm{H}), 3.76\left(\mathrm{~s}, 8 \mathrm{H}, 4 \times \mathrm{OCH}_{2}\right), 3.68(\mathrm{~s}, 4 \mathrm{H}$, $\left.2 \times \mathrm{NCH}_{2}\right), 3.59\left(\mathrm{~s}, 4 \mathrm{H}, 2 \times \mathrm{NCH}_{2}\right)$; HRMS (ESI) calcd for $\mathrm{C}_{25} \mathrm{H}_{25} \mathrm{~N}_{2} \mathrm{O}_{9}[\mathrm{M}+\mathrm{H}]^{+}$497.1560, found 497.1553.

7,4'-二(哌啶- $N$-甲酰氧基)-5-羟基异黄酮 $(\mathbf{8 f})$ : 类白 色固体，产率 57.7\%. m.p. $230 \sim 231{ }^{\circ} \mathrm{C} ;{ }^{1} \mathrm{H}$ NMR $\left(\mathrm{CDCl}_{3}, 400 \mathrm{MHz}\right) \delta: 12.74(\mathrm{~s}, 1 \mathrm{H}, 5-\mathrm{OH}), 7.95(\mathrm{~s}, 1 \mathrm{H}$, 2-H), 7.52 (d, $\left.J=8.4 \mathrm{~Hz}, 2 \mathrm{H}, 2^{\prime}, 66^{\prime}-\mathrm{H}\right), 7.20$ (d, $J=8.4 \mathrm{~Hz}$, $\left.2 \mathrm{H}, 3^{\prime}, 5^{\prime}-\mathrm{H}\right), 6.81$ (d, $\left.J=2.0 \mathrm{~Hz}, 1 \mathrm{H}, 6-\mathrm{H}\right), 6.60$ (d, $J=2.0$ $\mathrm{Hz}, 1 \mathrm{H}, 8-\mathrm{H}$ ), 3.60 (brs, 4H, $2 \times \mathrm{NCH}_{2}$ ), 3.53 (brs, 4H, $2 \times \mathrm{NCH}_{2}$ ), 1.62 (brs, 12H, $6 \times \mathrm{CH}_{2}$ ); HRMS (ESI) calcd for $\mathrm{C}_{27} \mathrm{H}_{29} \mathrm{~N}_{2} \mathrm{O}_{7}[\mathrm{M}+\mathrm{H}]^{+}$493.1975, found 493.1968.

7,4'-二(4-甲基哌嗪- $N$-甲酰氧基)-5-羊基异黄酮(8g): 类白色固体，产率 $52.0 \%$. m.p. $223 \sim 225{ }^{\circ} \mathrm{C} ;{ }^{1} \mathrm{H}$ NMR $\left(\mathrm{CDCl}_{3}, 400 \mathrm{MHz}\right) \delta: 12.74(\mathrm{~s}, 1 \mathrm{H}, 5-\mathrm{OH}), 7.95(\mathrm{~s}, 1 \mathrm{H}$, 2-H), 7.53 (d, $\left.J=8.4 \mathrm{~Hz}, 2 \mathrm{H}, 2^{\prime}, 6{ }^{\prime}-\mathrm{H}\right), 7.20$ (d, $J=8.4 \mathrm{~Hz}$, $\left.2 \mathrm{H}, 3^{\prime}, 5^{\prime}-\mathrm{H}\right), 6.81$ (d, $\left.J=2.0 \mathrm{~Hz}, 1 \mathrm{H}, 6-\mathrm{H}\right), 6.61$ (d, $J=2.0$ $\mathrm{Hz}, 1 \mathrm{H}, 8-\mathrm{H}$ ), 3.71 (brs, 4H, $2 \times \mathrm{NCH}_{2}$ ), 3.62 (brs, 4H, $\left.2 \times \mathrm{NCH}_{2}\right), \quad 2.50$ (brs, $\left.8 \mathrm{H}, \quad 4 \times \mathrm{NCH}_{2}\right), 2.37(\mathrm{~s}, \quad 6 \mathrm{H}$, $2 \times \mathrm{CH}_{3}$ ); HRMS (ESI) calcd for $\mathrm{C}_{27} \mathrm{H}_{31} \mathrm{~N}_{4} \mathrm{O}_{7}[\mathrm{M}+\mathrm{H}]^{+}$ 23.2193, found 523.2194.

7,4'-二(4-芳基哌嗪- $N$-甲酰氧基)-5-羊基异黄酮(8h): 类白色固体，产率 $67.8 \%$. m.p. $213 \sim 215{ }^{\circ} \mathrm{C} ;{ }^{1} \mathrm{H}$ NMR $\left(\mathrm{CDCl}_{3}, 400 \mathrm{MHz}\right) \delta: 12.74(\mathrm{~s}, 1 \mathrm{H}, 5-\mathrm{OH}), 7.95(\mathrm{~s}, 1 \mathrm{H}$, 2-H), 7.52 (d, J=8.4 Hz, 2H, 2',6'-H), 7.35 $7.29(\mathrm{~m}$, $10 \mathrm{H}, \mathrm{ArH}), 7.19$ (d, $\left.J=8.4 \mathrm{~Hz}, 2 \mathrm{H}, 3^{\prime}, 5^{\prime}-\mathrm{H}\right), 6.80$ (d, $J=$ $2.0 \mathrm{~Hz}, 1 \mathrm{H}, 6-\mathrm{H}), 6.60$ (d, $J=2.0 \mathrm{~Hz}, 1 \mathrm{H}, 8-\mathrm{H}), 3.69$ (s, $\left.4 \mathrm{H}, 2 \times \mathrm{NCH}_{2}\right), 3.59\left(\mathrm{~s}, 8 \mathrm{H}, 4 \times \mathrm{NCH}_{2}\right), 2.53(\mathrm{~s}, 8 \mathrm{H}$, $4 \times \mathrm{NCH}_{2}$ ); HRMS (ESI) calcd for $\mathrm{C}_{39} \mathrm{H}_{39} \mathrm{~N}_{4} \mathrm{O}_{7}[\mathrm{M}+\mathrm{H}]^{+}$ 675.2819, found 675.2814.

\subsection{AChE 及 BuChE 抑制活性测定}

向 96 孔板中依次加入 $1.0 \mathrm{mmol} / \mathrm{L}$ 碘化硫代乙酰胆 碱或碘化硫代丁酰胆碱(均购自 Sigma 公司) $30 \mu \mathrm{L}, \mathrm{pH}$ 7.4 的 PBS 缓冲液 $40 \mu \mathrm{L}$ 、待测化合物溶液 $20 \mu \mathrm{L}$ (DMSO 含量小于 $1 \%$ ) 和 $10 \mu \mathrm{L}$ 乙酰胆碱酯酶(大鼠脑皮层 $5 \%$ 匀 浆上清液, $\mathrm{pH} 7.4$ 的磷酸缓冲液作匀浆介质)或丁酰胆碱 酯酶(大鼠血清 $25 \%$ 上清液, $\mathrm{pH} 7.4$ 磷酸缓冲液作匀浆介 质)溶液, 加毕混匀后, $37{ }^{\circ} \mathrm{C}$ 孵育 $15 \mathrm{~min}$, 向各孔中加入 质量分数为 $0.2 \%$ 的 $5,5^{\prime}$-二硫代-双(2-硝基苯甲 酸)(DTNB，购自 Sigma 公司)溶液 $30 \mu \mathrm{L}$ 显色，用酶标仪 
测定 $405 \mathrm{~nm}$ 处各孔的光密度( $\mathrm{OD}$ 值), 与不加待测样品 的空白孔比较, 计算化合物对酶的抑制率 [酶抑制率 $=$ (1一样品组 OD 值/空白组 OD 值) $\times 100 \%$; 选择化合物 的五至六个浓度, 测定其酶抑制率, 并以该化合物摩尔 浓度的负对数与酶的抑制率线性回归, 求得 $50 \%$ 抑制率 时的摩尔浓度即为该化合物的 $\mathrm{IC}_{50}$.

\section{9 对 $\mathrm{H}_{2} \mathrm{O}_{2}$ 诱导的 PC12 细胞损伤的保护作用测定}

PC12 细胞用含 $10 \%$ 小牛血清的 DMEM 培养液 (Sigma 公司), 以 $1 \times 10^{5}$ 个 $/ \mathrm{mL}$ 密度接种于 96 孔培养板 上, 接种体积为 $100 \mu \mathrm{L} /$ 孔, 随后放入含 $5 \% \mathrm{CO}_{2}$ 的 $37{ }^{\circ} \mathrm{C}$ 恒温培养箱内培养; 培养 $24 \mathrm{~h}$ 后, 给药组中加相应浓度 的化合物(终浓度为 $10^{-5} \mathrm{~mol} / \mathrm{L}$ ) $10 \mu \mathrm{L} /$ 孔, 预粰育 $2 \mathrm{~h}$ (对 照组与损伤组分别加 $10 \mu \mathrm{L} /$ 孔 PBS); PC12 细胞捊育 $2 \mathrm{~h}$ 后, 在给药组与损伤组中分别加入 $100 \mu \mathrm{mol} / \mathrm{L} \mathrm{H}_{2} \mathrm{O}_{2}$ 损 伤剂 $10 \mu \mathrm{L} /$ 孔(对照组加 $10 \mu \mathrm{L}$ 孔 PBS), $30 \mathrm{~min}$ 后, 将各 组的培养液均换成无小牛血清无酚红的 DMEM 培养液 (Sigma 公司)继续放入恒温培养箱内培养 $24 \mathrm{~h}$, 然后各 组中加入 $5 \mathrm{mg} / \mathrm{mL}$ 的 3-(4,5-二甲基噻唑-2-基)-2,5-二苯 基四氮唑溴盐(MTT) $100 \mu \mathrm{L} /$ 孔，进行活细胞染色, $3 \mathrm{~h}$ 后, 各组中加入 $100 \% \mathrm{DMSO}$ 终止液 $100 \mu \mathrm{L} /$ 孔, 充分溶 解混匀, 在 $570 \mathrm{~nm}$ 的波长下测定各组的 OD 值, 以对照 组为 $100 \%$, 给药组及损伤组值以对照组的百分比表示.

\section{References}

[1] Liu, R. T.; Lu, Q. J. Acta Pharm. Sin. 2009, 44, 258 (in Chinese). (刘睿婷, 吕秋军, 药学学报, 2009, 44, 258)

[2] Mehta, M.; Adem, A.; Sabbagh, M. Inter. J. Alzheimer's Disease 2012, DIO: $10.1155 / 2012 / 728983$.

[3] Cavalli, A.; Bolognesi, M. L.; Minarini, A.; Rosini, M.; Tumiatti, V.; Recanatini, M.; Melchiorre C. J. Med. Chem. 2008, 51, 347.

[4] Fang, L.; Graus, B.; Lehmann, J.; Heilmann, J.; Zhang, Y.; Decker, M. Bioorg. Med. Chem. Lett., 2008, 18, 2905.

[5] Bang, O. Y.; Hong, H. S.; Kim, D.H.; Kim, H.; Boo, J. H.; Huh, H.; Mookjung, I. Neurobiol. Dis. 2004, 16, 21.

[6] Andlauer, W.; Kolb, J.; Furst, P. J. Nutr. 2000, 130, 3021.

[7] Lewis, P. T.; Wähälä, K.; Hoikkala, A.; Mutikainen, I.; Meng, Q. H.; Adlercreutz, H.; Tikkanen, M. J. Tetrahedron 2000, 56, 7805.

[8] Lu, J. R.; Xing, H.; Long, W. Y.; Li, Y. M.; Yang, Y.; Huang, W. L. Chin. J. Org. Chem. 2011, 31, 1884 (in Chinese). (芦金荣, 邢辉, 龙文艳, 李运曼, 杨显, 黄文龙, 有机化学, 2011, 31, 1884)

[9] Cheng, D. H.; Tang, X. C. Pharmacol., Biochem. Behav. 1998, 60, 377.

[10] Zhang, J.; Zhu, D. F.; Sheng, R.; Wu, H. H.; Hu, Y. Z.; Wang, F.; Cai, T.; Y.; Yang, B.; He, Q. J. Eur. J. Pharmacol. 2009, 613, 1.

[11] Adeppa, D.; Rupainwar, D. C.; Misra, K. Synth. Commun. 2011, $41,285$.

[12] Birkofer, L.; Krebs, K. Tetrahedron Lett. 1968, 7, 885.

[13] Hoppe, D.; Hanko, R.; Bronneke, A. Chem. Ber. 1985, 118, 2822.

[14] Hoshinoa, O.; Saitoa, K.; Ishizakia, M. Synth. Commun. 1987, 17, 1887.

[15] Morren, H.; Denayer, R. Bull. Soc. Chim. Belg. 1949, 58, 103.

[16] Lemoucheux, L.; Rouden, J.; Ibazizene, M.; Sobrio, F.; Lasne, M. C. J. Org. Chem. 2003, 68, 7289 . 